\title{
Gradhiva
}

GRADHIV

Revue d'anthropologie et d'histoire des arts

$25 \mid 2017$

Gottfried Semper, habiter la couleur

\section{Gottfried Semper et la morphologie : transformations, culture et symbole}

Gottfried Semper and morphology: transformations, culture and symbol

\section{Muriel van Vliet}

\section{(2) OpenEdition \\ 1 Journals}

Édition électronique

URL : http://journals.openedition.org/gradhiva/3404

DOI : 10.4000/gradhiva.3404

ISSN : 1760-849X

Éditeur

Musée du quai Branly Jacques Chirac

Édition imprimée

Date de publication : 31 mai 2017

Pagination : 152-177

ISBN : 978-2-35744-095-1

ISSN : 0764-8928

Référence électronique

Muriel van Vliet, «Gottfried Semper et la morphologie : transformations, culture et symbole », Gradhiva [En ligne], 25 | 2017, mis en ligne le 31 mai 2019, consulté le 03 janvier 2020. URL : http://

journals.openedition.org/gradhiva/3404; DOI : 10.4000/gradhiva.3404

(c) musée du quai Branly 


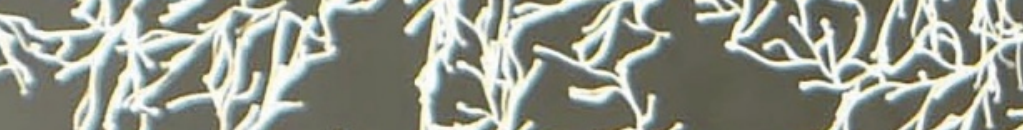

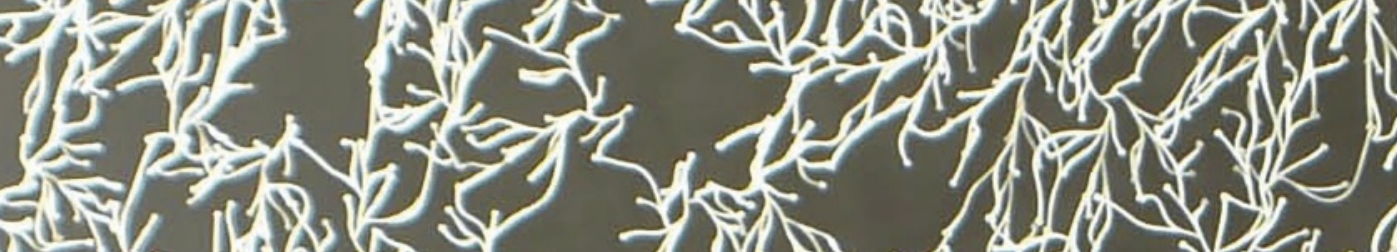
政

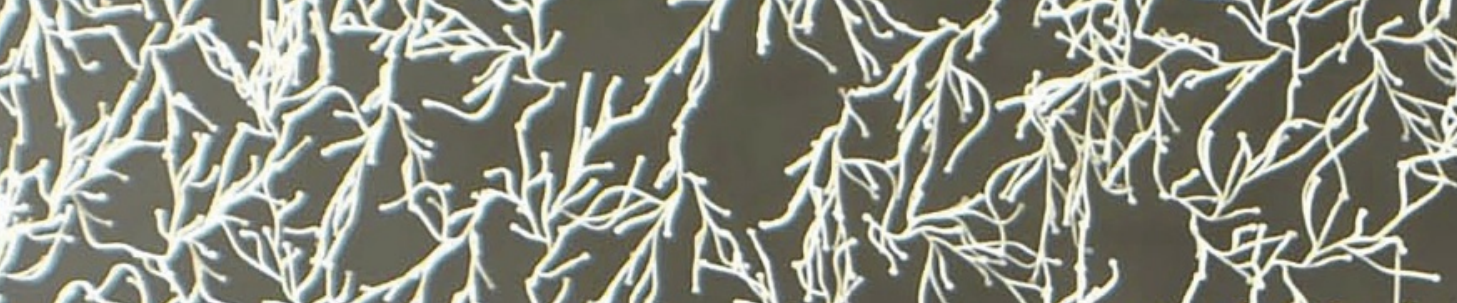

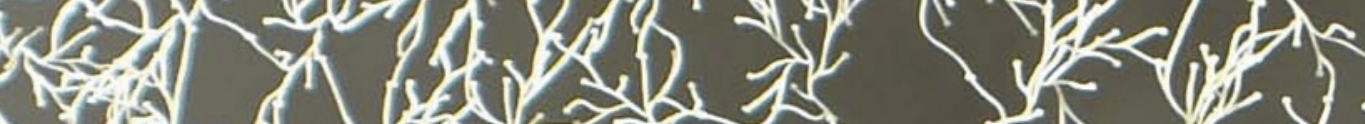

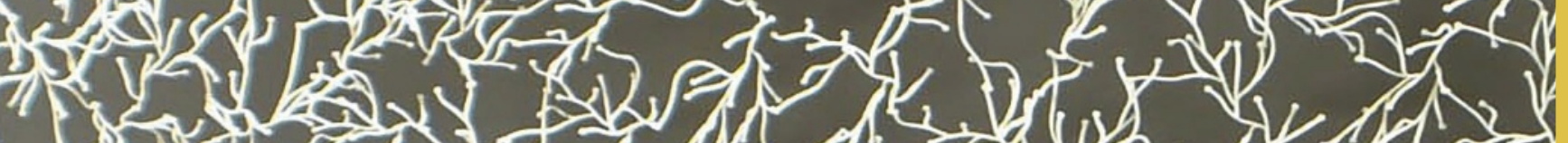

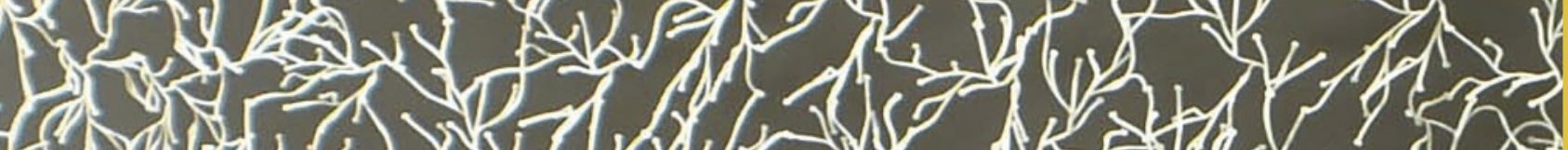

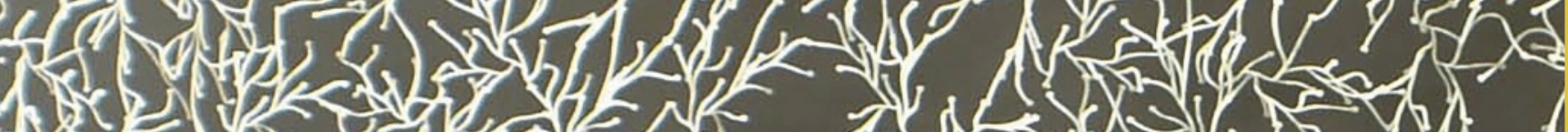
,

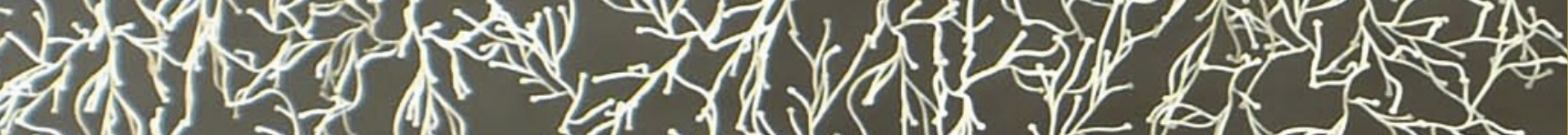
,

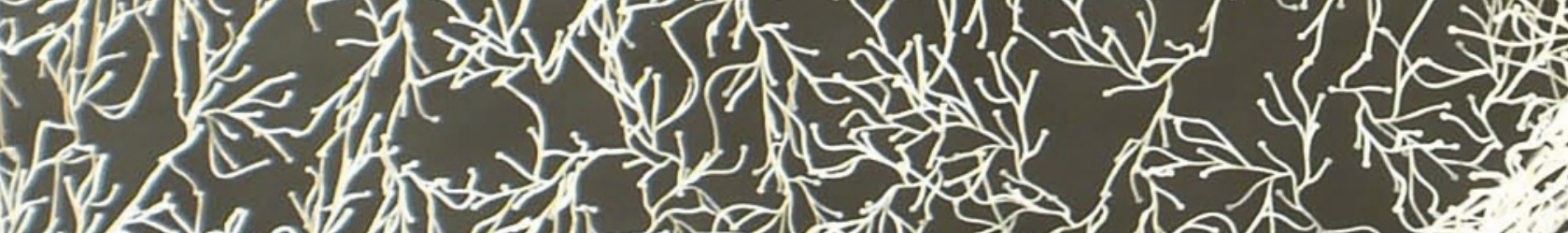
2.

, $42-1,1$

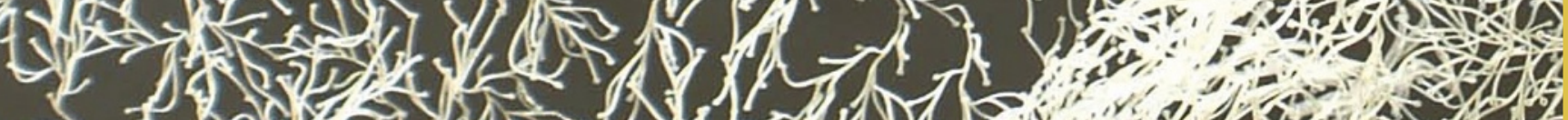

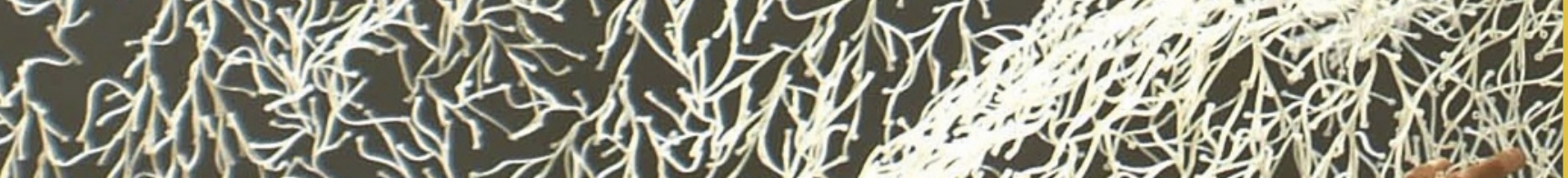

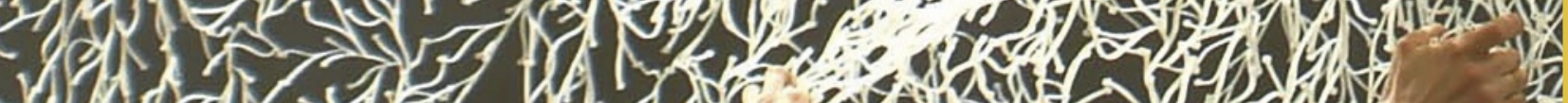
$13 / 20$,

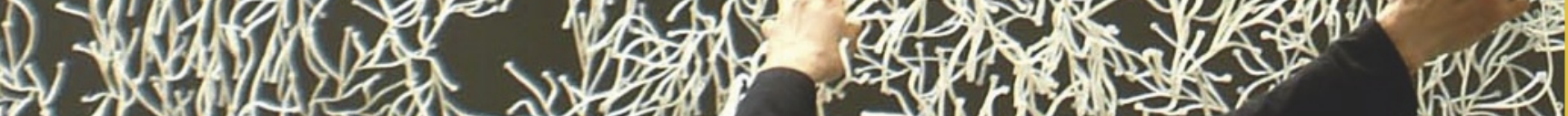

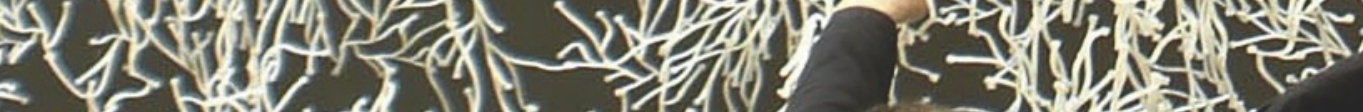
(2)

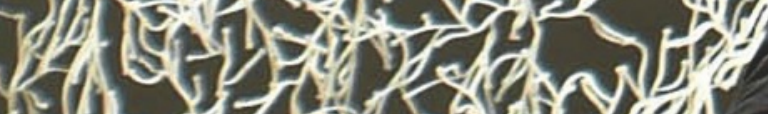
a (4) 10.10 


\section{Gottfiried Semper et la morphologie: transformations, culture et symbole}

par Muriel van Vliet

Gottfried Semper s'inscrit au sein d'une ligne morphologique née des écrits de Johann Wolfgang von Goethe et de Wilhelm von Humboldt. C'est à partir de la théorie de la culture qu'il aborde la question de la plasticité des formes. Mais Semper se heurte à un sentiment de crise qui fait toute la modernité de son œuvre par rapport à ses prédécesseurs. Sa réflexion sur la «tragédie» d'un individu soudain incapable de se frayer un chemin au sein de la culture est prolongée par Georg Simmel. Semper conçoit de manière originale l'ornementation comme symbole, véritable incarnation du mouvement du corps dans l'espace à la fois cosmique et social: c'est ce qui fait la spécificité de son approche de la forme et sa manière de résoudre les tensions qui se jouent entre individu et culture. 


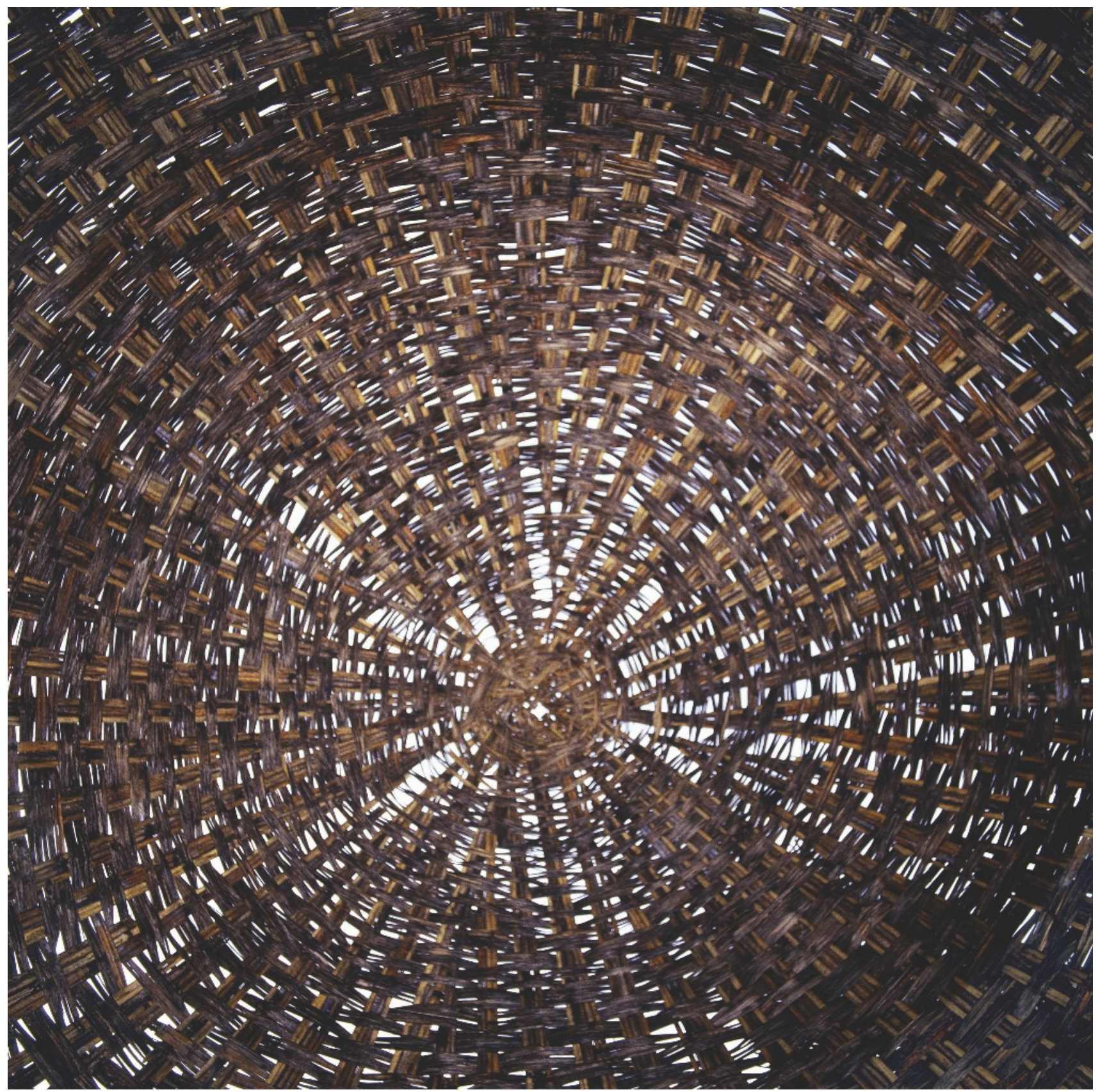


Gottfried Semper (1803-1879) pose la question de savoir ce qui rend une forme organique et vivante. Ce n'est pas au courant idéaliste d'un Emmanuel Kant ou d'un Georg Wilhelm Friedrich Hegel qu'il appartient, mais bien à cette ligne morphologique qui s'enracine dans les écrits de Johann Wolfgang von Goethe (1749-1832). Ce dernier définit la forme comme figure concrète, car ce qui l'intéresse est avant tout le processus de configuration permettant de créer des figures par transformation de cas prégnants. Si Goethe appartient à une génération antérieure, il est indéniable que Semper y trouve une de ses sources.

Par ailleurs, Semper connaît les écrits de Wilhelm von Humboldt (1767-1835), à la fois contemporain de Goethe et adversaire historique de Hegel. Le linguiste et anthropologue applique la méthode morphologique à l'étude du langage tout en inscrivant celle-ci dans une théorie générale de la culture. Semper prolonge de fait les intuitions de Humboldt du côté de l'art et de l'architecture, car c'est à partir d'une théorie de la culture élargie qu'il va pouvoir aborder la question de la plasticité des formes.

Semper se heurte toutefois à un sentiment de crise qui fait toute la modernité de son œuvre. Celle-ci se joue dans le rapport qu'entretient l'individu avec la culture et la société au moment de l'avènement de la reproductibilité technique. Georg Simmel (1858-1918) prolongera sa réflexion sur cette potentielle «tragédie» d'un individu se sentant soudain incapable de se frayer un chemin au sein de la culture et réfléchira comme lui aux questions pédagogiques d'exposition de la culture et de l'art.

Mais c'est grâce à la conception de l'ornementation comme symbole, c'est-à-dire comme incarnation du mouvement du corps dans l'espace tant cosmique que social, que cette crise va potentiellement trouver à se résoudre. De ce point de vue, la théorie sempérienne du style trouve des échos plus tardifs dans la théorie de l'homme en mouvement qu'esquissent respectivement l'historien de l'art Aby Warburg (1866-1929), le philosophe Ernst Cassirer (1874-1945), ainsi que le philosophe et historien de l'art Edgar Wind (1900-1971), trois auteurs liés à la ville de Hambourg, comme Semper.

En partant de l'architecture et en se centrant sur l'ornementation, Semper donne à l'approche morphologique une tonalité spécifique. Et c'est cette originalité que nous voudrions mettre progressivement en lumière, en faisant saillir par contrastes successifs la spécificité de son interprétation de la transformation des figures, de la culture et du symbole.

La théorie de l'ornementation annonce un structuralisme morphogénétique dont des auteurs tels que Claude Lévi-Strauss déploieront à leur manière les potentialités. L'étude de la postérité allemande de la morphologie goethéenne permet d'éclairer certains aspects du néostructuralisme.

\section{L'apport goethéen à la conception sempérienne de la figure et du style}

Dans son poème sur La Métamorphose des plantes (Goethe 2013), ses Écrits sur l'art (1996), mais aussi au travers de sa riche correspondance, ci-contre

fig. 1

Éthiopie, province de

Gamo Gofa, Chencha

Le peuple dorze vivant dans l'ouest de la vallée du Rift construit son habitat en bambou tressé. Photo (C) Nigel Pavitt, AWL Images / Getty images. 
1. Pour cette phrase de Goethe ainsi que la précédente voir: 1887-1919, "Von deutscher Baukunst " in Goethes Werke, t. XXXVII, Sophie von Sachsen (éd.), 4 sections, 133 volumes. Weimar: 148, cité par Ernst Cassirer 1975: 201-202

2. Le type originaire n'est progressivement dit tel par Goethe qu'au sens où il est fondamental ou élémentaire et qu'il permet de condenser le principe en lui-même idéel d'une mise en série d'éléments.
3. Voir Cassirer 2001, "Goethe » : 238: «C'est la méthode spécifique de la "variation", qu'elle se réfère à une expérience scientifique ou à un motif poétique, c'est la "dérivation" à partir d'un "point marquant" efficace et fécond dans les deux cas qui constitue pour Goethe l'opposition décisive entre les hommes empiriques, simplement réceptifs, et l'homme créateur. "

4. Voir "Simple imitation de la nature, manière, style (1789) ", Goethe 1996

5. Aloïs Riegl (1858-1905) s'opposera à son tour à ce qu'il désignera comme l'évolutionnisme matérialiste de Semper (et d'Adolf Hildebrand). II faut selon Riegl s'intéresser à l'évolution stylistique, soutenue par une pulsion artistique immanente, qu'il retraduira comme volonté d'art (Kunstwollen). Voir Riegl 2002 [1893] ainsi que le commentaire de François-René Martin, "Aloïs Riegl. Questions de style: fondements d'une histoire de l'ornementation ", Critique d'art 21 (1), 2003 [en ligne], disponible sur http://critiquedart.revues org/1952 (consulté le 18 janvier 2017).

6. Voir Goethe 1986. L'intérêt de Semper pour la polychromie peut s'inscrire dans le droit fil de cet ouvrage de Goethe.
Goethe n'apparaît pas seulement comme un poète, mais également comme un penseur de la forme, qu'il redéfinit comme figure. Animée par un processus actif de configuration, la figure est selon lui essentiellement plastique. Le cœur de l'approche esthétique de Goethe n'est d'ailleurs plus tant la beauté que ce processus permanent de reconfiguration: «L'art est formateur bien avant d'être beau [...]. Car l'homme possède en lui une nature formatrice qui se manifeste dès que son existence est assurée. " La beauté des œuvres d'art n'a de valeur que parce qu'elle témoigne d'un processus de formation, attestant de la liberté de création d'un individu qui a su progressivement s'élever de la simple imitation de la nature au niveau du style (Goethe 1996). L'humanisme de Goethe ouvre de plus à un intérêt nouveau, bien qu'encore discret, pour les «cocos et les plumes ${ }^{1}$ » des peuples non-occidentaux. Là encore, ce qui compte à ses yeux, ce n'est plus dans l'absolu la beauté ou la laideur mais le processus qui, de manière toujours relative, forme et informe. II s'agit avant tout de différencier diverses manières de procéder à la mise en figure.

La configuration se définit comme transformation à partir d'un type originaire $^{2}$. Elle correspond à la dérivation d'une série à partir d'un cas prégnant que l'on fait librement varier ${ }^{3}$. La configuration témoigne d'un rythme oscillatoire, diastole et systole harmonisant repos et mouvement de la figure. Or ce processus caractérise tout autant l'organisme vivant que l'art. Goethe refuse en effet toute coupure ou opposition entre organisme naturel et $œ u v r e$ culturelle. L'œuvre culturelle redouble le processus qui rend vivant l'organisme naturel, lui-même susceptible d'évolution. Cependant, malgré cette approche croisée du vivant et de l'art, Goethe ne prône pas l'imitation de la nature par l'art. II ne défend pas non plus une conception naturalisante de la culture. Si les formes humaines peuvent être à juste titre rapprochées des organismes naturels vivants, ce n'est jamais pour réduire la production culturelle à un prétendu mécanisme naturel, dont le modèle est à rejeter. Selon Goethe, la nécessité intérieure qui anime toute forme n'est en rien exclusive de la liberté créatrice de l'imagination humaine qui procède à diverses expérimentations libres. Contre tout déterminisme, il défend une conception résolument humaniste de l'homme, véritable second Prométhée. L'art doit s'élever téléologiquement de la simple imitation mécanique de la nature, jusqu'au moment caractérisé par ce qu'il nomme la «manière», encore trop subjective, puis à celui du style ${ }^{4}$. Le style correspond au niveau où l'individu créateur et les figures créées entrent en résonance au travers d'une série d'expérimentations qui en dégagent l'essence.

Cette position morphologique dynamique réagit contre un formalisme abstrait qui couperait les formes tout autant du processus qui les a vues naître que du matériau et des techniques utilisés ou encore du milieu environnant ${ }^{5}$. Le matériau se trouve valorisé, avec par exemple des études sur la couleur, dont Goethe explore le phénomène d'organisation dans son Traité des couleurs ${ }^{6}$. Les figures doivent être replacées dans la globalité d'une expérience où la figure interagit avec l'environnement sans rien perdre pour autant de son autonomie. Goethe ne néglige jamais l'importance de l'atmosphère (Stimmung) que cet environnement global dégage, sa tonalité propre. 
Or, selon Semper, tout comme pour Goethe, «chaque forme, refermée sur soi, s'associe pour ainsi dire à une matérialité auprès de laquelle le devenir forme (Gestaltung) et la conservation de soi constituent des forces actives ${ }^{7}$ » (Semper 2007: 252). Du conflit entre les forces de gravitation, de vie et de volonté «doit résulter une transformation de la forme qui fait que ce qui vit accède à l'existence » (ibid.).

On perçoit en outre l'importance de la lecture de Goethe lorsque Semper définit la notion centrale de style:

Le style, c'est la mise en évidence, élevée jusqu'à l'expression artistique, de l'idée originaire et de tous les facteurs intérieurs et extérieurs qui influent, en la modifiant, sur la mise en forme matérielle qu'ils ont contribué à établir. [...] De même que la nature, avec toute sa diversité, se montre toujours économe et simple en ses motifs, de même qu'elle ne montre qu'un renouvellement constant des mêmes formes mille fois modifiées suivant l'échelle graduée du perfectionnement et les diverses conditions d'existence des êtres [...], de même il existe un ensemble de formes originaires servant de base aux arts techniques et qui, quoiqu'imposées par une idée primitive, permettent cependant, malgré leur réapparition constante, une diversité infinie commandée par des conditions qu'imposent des circonstances particulières et précises ${ }^{8}$. (Semper 2012: 69)

Le style témoigne de la libre capacité de créer une nouvelle série originale à partir de ce qui est déjà inclus dans une série d'ordre inférieur dont le principe est au commencement guidé par des besoins naturels universels fondamentaux, autrement dit des dispositifs biologiques.

Pour définir le pouvoir créateur de l'artiste, Goethe utilise la métaphore du kaléidoscope ${ }^{9}$, objet récemment inventé à son époque, que l'on tourne jusqu'à faire naître des figures nouvelles. Par dérivation imaginative réglée, l'artiste est capable par exemple, à partir du cas prégnant que constitue un vitrail d'église particulièrement complexe, de faire surgir à l'infini de nouvelles figures:

J'avais ce don, nous dit Goethe, quand je fermais les yeux, tête basse, pour me représenter une fleur au centre de mon organe de vue, que celle-ci ne se figeait pas un seul instant sous sa forme première, mais s'ouvrait et dans son centre s'épanouissaient de nouvelles fleurs aux pétales colorés et feuilles vertes; ce n'étaient pas des fleurs naturelles, mais des fleurs imaginaires, bien que régulières comme les rosaces d'un sculpteur. II était impossible de fixer cette création jaillissante, elle durait en revanche aussi longtemps que je le désirais, sans gagner ni perdre de sa force. Je pouvais de même, quand je me représentais un vitrail, faire naître son décor polychrome qui ensuite se transformait sans cesse depuis le centre jusqu'à la périphérie, à l'image du kaléidoscope récemment inventé ${ }^{10}$. (Semper 2012 : 69)

Or Semper emploie à maintes reprises la notion goethéenne de « dérivation », permettant de «faire ressortir par elles-mêmes les formes de leurs
7. Ce sera un des nombreux points sur lesquels Aloïs Riegl s'opposera à Semper. Deux interprétations de I'héritage morphologique se trouvent alors confrontées.

8. Nous soulignons.

9. Goethe, «Das Sehen in subjektiver Hinsicht ", cité et commenté par Cassirer 2001: 233.

10. Nous soulignons. On retrouvera cette métaphore sous la plume de Lévi-Strauss 1962 : 51-52 dans un célèbre passage: "Cette logique [requise par les sciences humaines] opère un peu à la façon du kaléidoscope: instrument qui contient aussi des bribes et des morceaux, au moyen desquels se réalisent des arrangements structuraux." 

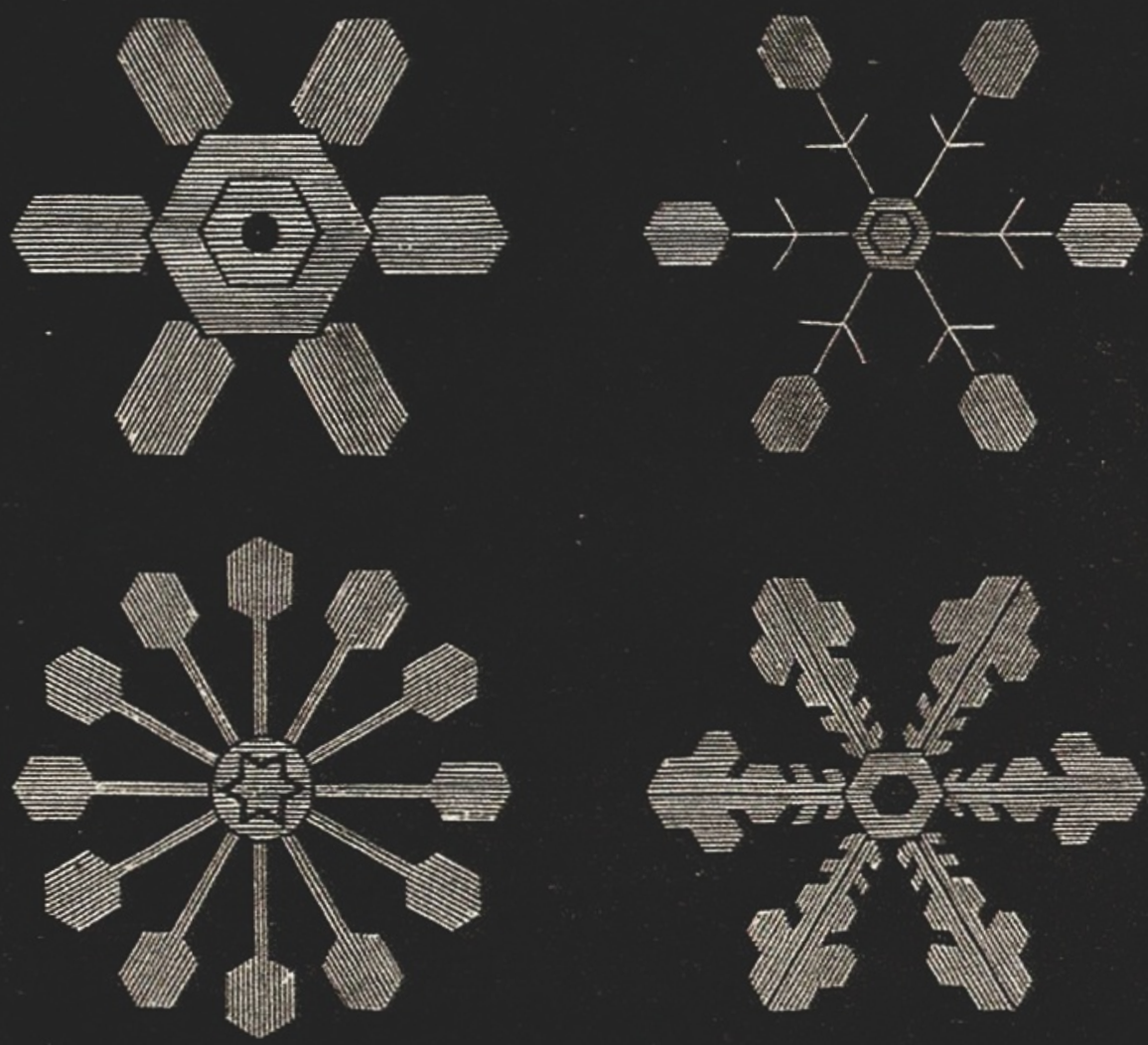

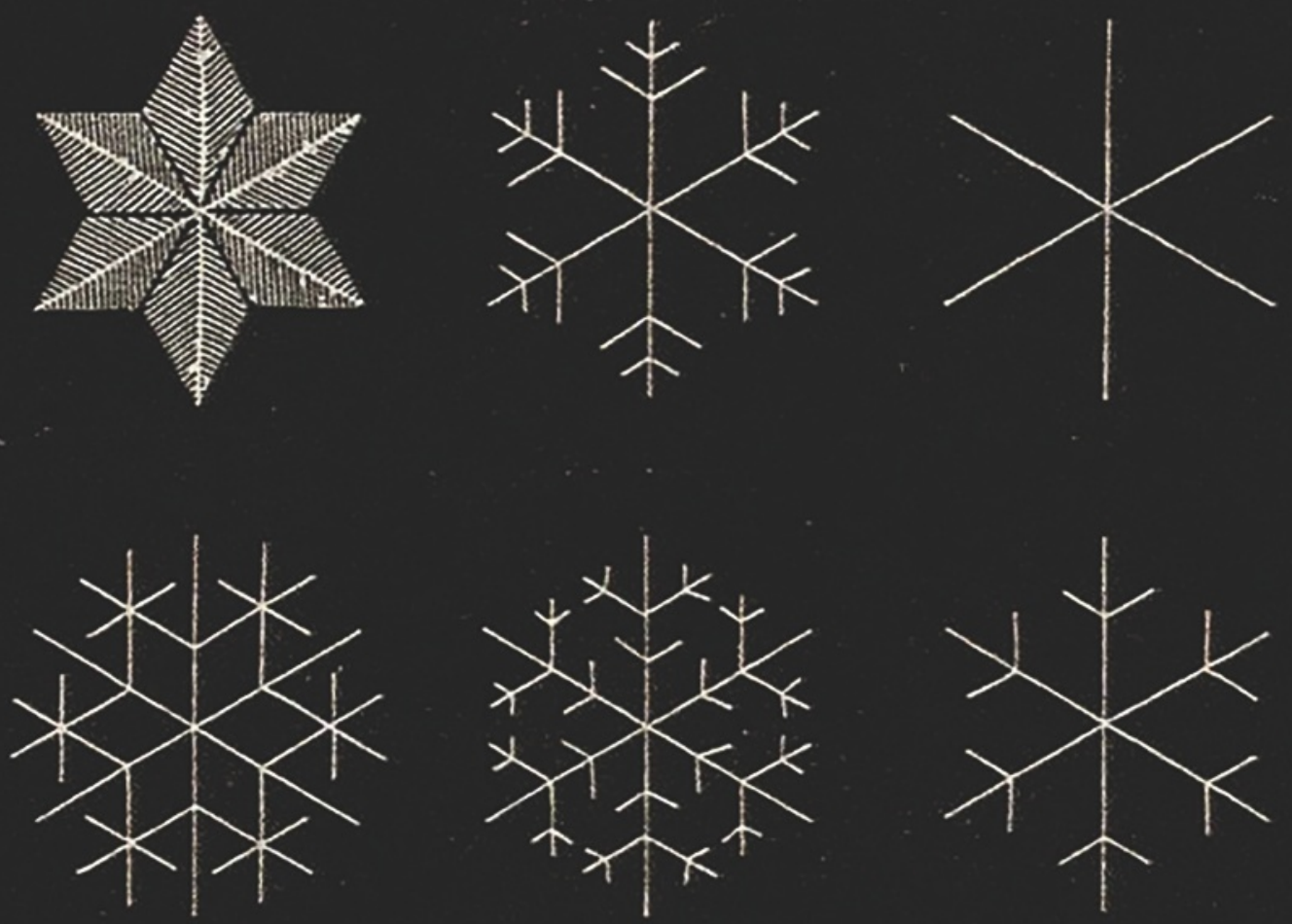

fig. 2

Gottfried Semper,

"Flocons" in Der Stil, t. I

(éd. 1878), p. xxv-xxvi. 
11. Nous soulignons

12. Voir Goethe 2013. Le vers allemand " Und so deutet das Chor auf ein geheimnes Gesetz » et celui qui le précède peuvent être traduits ainsi : "Toutes ces formes se ressemblent et aucune à l'autre n'est pareille. Et c'est pourquoi leur chœur suggère à notre esprit une loi secrète» (Goethe 1982).

13. "Beauté, qui est moins une propriété de l'œuvre qu'un effet en vertu duquel les composantes très variées, extérieures et intérieures de l'objet auquel le prédicat beau s'applique, agissent de concert » (nous soulignons).

14. Voir Semper 2007 "cœur caché": 63; "chef de chœur »: 255 et 298 ; "série»: 289 formes typiques primitives» pour obtenir des "variations de leur style» (LéviStrauss 1962: 61), tout en se penchant sur la dynamique de la polychromie. Lisons encore ce passage de Semper, en écho à Goethe:

Que si un motif artistique a subi un traitement matériel quelconque, son type originel sera alors modifié par ce traitement, prenant grâce à lui une tonalité (Stimmung) déterminée. Une métamorphose plus ou moins prononcée s'étant opérée en lui, le type abandonne ce qui pour ainsi dire correspond aux premières étapes de son développement. Que maintenant, à partir de cette transformation secondaire ou effectuée de multiples manières, il subisse un nouveau changement de matériau, alors la forme qui en résulte sera un résultat mélangé, exprimant à la fois l'archétype et toutes les étapes qui précèdent la dernière formation. Grâce à la reconstitution du bon déroulement de l'évolution, on pourra ainsi reconnaître l'ordre des chaînons intermédiaires qui font le lien entre l'expression primitive de l'idée artistique et ses multiples dérivés ${ }^{11}$. (Semper 2007: 334)

Malgré leur infinie diversité, les œuvres forment selon Goethe entre elles comme un "chœur caché ${ }^{12}$ ", une métaphore extraite du poème sur la métamorphose des plantes que l'on retrouve en de multiples occurrences chez Semper, non sans quelques accents leibniziens (ibid.: 253). Le lien entre musique et architecture se soude autour de la redéfinition du beau comme résultant d'une «action d'ensemble» (ibid.: 269) permettant de créer un effet de concert ${ }^{13}$ (ibid. : 284).

Certaines figures clés, voire certains arts clés, jouent d'ailleurs dans la combinatoire de Semper le rôle de "chef de chœur ", permettant d'harmoniser des séries de différents ordres, reliant ainsi indéfectiblement des figures qui semblaient présenter pourtant à première vue une hétérogénéité irréductible ${ }^{\mathbf{1 4}}$. Elles forment un «foyer idéal» (ibid.: 255).

Or les séries ne s'ordonnent pas tant de manière linéaire que selon un principe radial, mis en exergue par certaines figures primitives, tels le flocon ou le cristal (ibid.: 286). L'ordre se construit de manière dynamique, en fonction d'un jeu de forces simultanément centrifuge et centripète. Autrement dit, l'ordre se construit à la fois du centre vers la périphérie et de la périphérie vers le centre, selon des mouvements oscillatoires produisant un effet d'alternance entre repos et mouvement. L'ordre est une forme en devenir dont la plasticité est la caractéristique essentielle. "Se transporter mentalement au centre du réseau des relations " (ibid.: 287) permet à l'observateur de se rendre sensible à l'eurythmie des figures.

Lorsque l'on enquête sur le processus de formation du sens, les figures originaires, ou archétypes, sont les éléments en deçà desquels on ne peut remonter, mais également ceux dont il nous faut toujours repartir pour comprendre ce processus dans sa globalité. Et l'un des présupposés de Semper consiste précisément à défendre que c'est seulement en étant abordé dans sa globalité que le phénomène de formation peut vraiment être compris. Ils sont donc à dénombrer et à décrire comme autant de principes productifs, stimulant la création à venir. L'approche morphologique n'est à ce titre pas tant 
fondation a priori comme chez Kant que description minutieuse du fonctionnement de l'expérience des formes, enquête en droit inachevable, ouverte sur la création à venir.

Il faut ajouter à ceci que les formes originaires ne sont pas nécessairement simples, elles peuvent même être dites stricto sensu «complexes", au sens où on peut considérer rétrospectivement qu'elles indiquent les directions selon lesquelles peuvent se réaliser des déploiements variés. Elles condensent les traits caractéristiques qui vont se déployer différemment ou même séparément dans diverses figures dérivées. Semper note bien ce point. La mise en ordre ne va pas de manière analytique et linéaire du simple au complexe, comme le présenterait un idéaliste tel René Descartes, mais bien du complexe, comme matrice, au simple, que l'on peut en dériver. L'approche est en quelque sorte «holiste».

On retrouve chez Semper ce principe de la radialité de la mise en série (ibid.: 243). Le principe de radialité permet d'attirer l'attention sur un point de référence central autour duquel un ensemble se trouve arrangé (ibid.). Comme nous venons de le rappeler, l'architecture apparaît comme le chef d'orchestre des arts appliqués, qui se projettent sur l'aire relationnelle qu'elle constitue, en y trouvant leur meilleur lieu d'expression. Ils y révèlent en effet le passage possible d'un stade plus mécanique, commandé essentiellement par les besoins primordiaux, à une compréhension expressive, par laquelle l'homme réalise la liberté qu'il peut prendre par rapport au matériau choisi et l'écart que sa production présente par rapport aux formes originaires, liées étroitement encore à des fonctions biologiques. L'architecte est celui qui a conscience de la beauté qu'il y a à pouvoir conserver ces formes originaires nées des différents arts appliqués (et donc des besoins primaires), tout en les transformant radicalement par l'acquisition d'un style. Une certaine autonomie se trouve conquise par rapport aux formes et au matériau, sans que soient pour autant perdues de vue l'histoire de ces formes et la fonction de ce matériau. La force d'une architecture va tenir à la maîtrise de ce grand écart entre maintien de formes originaires et libération de ces formes.

Chez Semper, le style consiste dans la concordance d'un phénomène artistique avec l'histoire de sa formation, ainsi qu'avec toutes les conditions et circonstances de son devenir. C'est en quelque sorte la réflexion sur l'architecture et la projection des arts appliqués sur cette dernière, nés toutefois indépendamment d'elle et chacun selon leur logique propre, qui permet le passage, selon Semper, de la simple imitation de la nature à l'expression libre et au style.

\section{Les apports humboldtiens à l'anthropologie de Semper}

Semper tire également de la lecture des écrits de Humboldt certains points décisifs pour sa propre analyse.

L'étude du chinois par Humboldt trouve son pendant chez Semper, fasciné quant à lui par les découvertes récentes des archéologues en Égypte et en Assyrie. Semper veut bousculer l'approche de l'univers grec, en montrant que ce pôle prégnant de l'histoire des arts dont le temple dorique condense 
Pl. 4

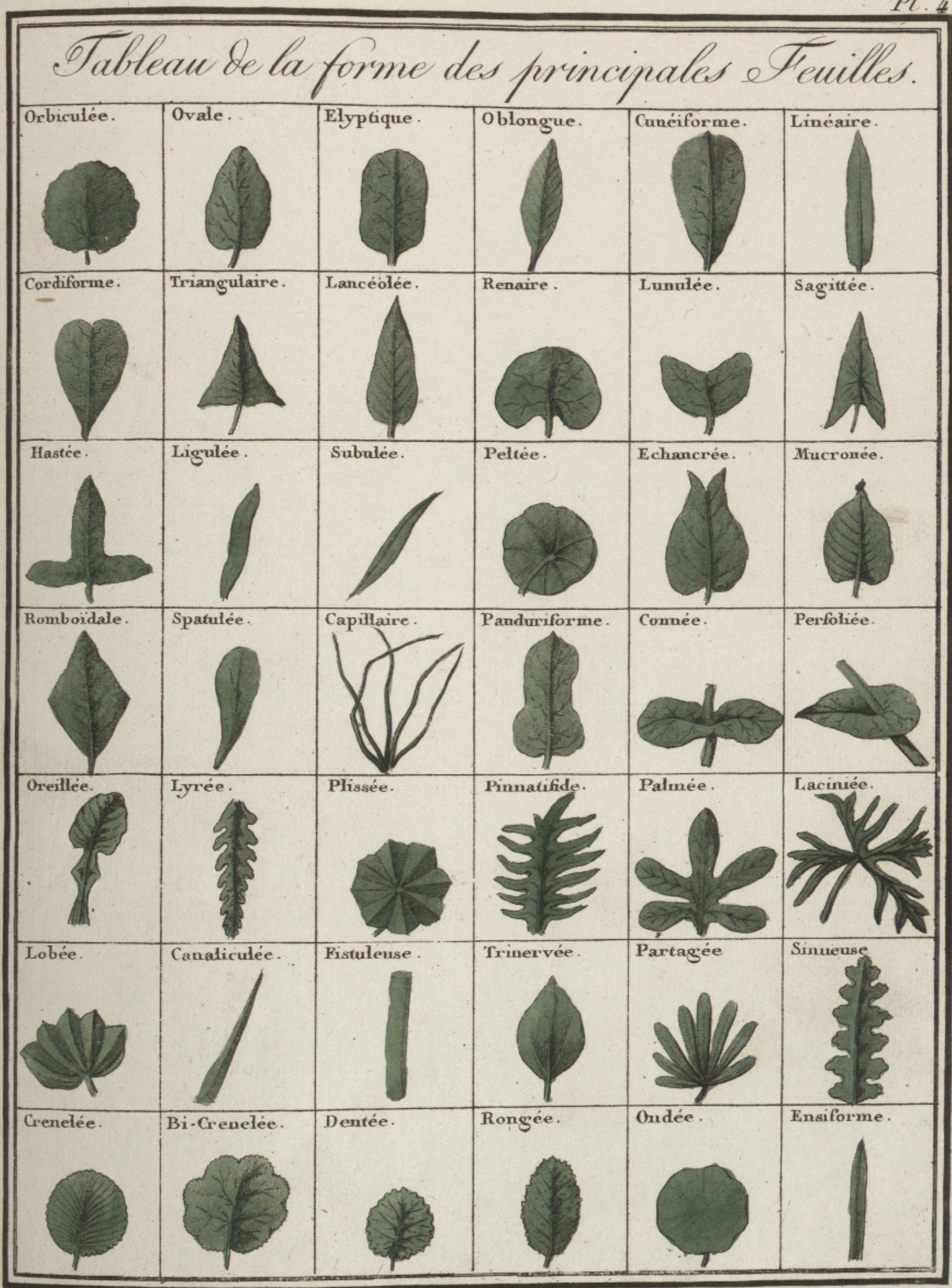

THEPUE 
les traits ne peut être bien compris qu'une fois replacé au centre d'un réseau multipolaire, incluant des formes égyptiennes et assyriennes. L'art grec se trouve soudainement abordé comme la libre reprise de formes «barbares», non conscientes encore des différents principes qui les régissent. Humboldt comme Semper créent chez les lecteurs contemporains un effet de dépaysement «baroque», bousculant la géographie habituelle qui privilégie la Grèce, idéalisée, et l'Europe.

En un sens, Humboldt transpose la morphologie goethéenne à l'étude des phénomènes langagiers qu'il entreprend de comparer. Les langues forment malgré leur grande et infinie diversité comme un «chœur caché ${ }^{15}$ », car on peut traduire une langue dans une autre et observer par quelles mutations les langues évoluent progressivement, tout en conservant chacune leur génie propre, irréductible. Et Semper élargit, pourrait-on dire, la conception humboldtienne du langage à l'approche de l'art en général, et de l'architecture en particulier.

Humboldt conçoit le langage non seulement comme un simple outil, un instrument, un ergon secondaire, annexe ou extérieur, mais aussi et surtout comme une energeia, une énergie, un champ dans lequel évolue la pensée comme dans son Denkraum, espace de pensée qui est aussi Lebensraum, espace de vie ${ }^{\mathbf{1 6}}$. Le langage est animé par une forme interne qui se déploie dans le dialogue compris comme processus d'interprétation et de réinterprétation, autrement dit comme traduction créatrice. La revalorisation par Semper des techniques et du matériau, voire des outils et de la main qui travaille, va elle aussi dans le sens d'une revalorisation du médium comme pivot essentiel. Les techniques ne sont, selon Semper, pas seulement ergon, mais aussi energeia: c'est par leur maîtrise que l'individu se forme en formant le monde qui l'entoure.

D'un point de vue politique et social, contre Hegel qui est son principal opposant, Humboldt défend une approche humaniste républicaine du rapport de l'individuel à l'universel: l'individu est et doit rester le point agissant de départ, même si son action créatrice consiste à réinterpréter des types généraux qui ont déjà une longue histoire et le font implicitement participer à l'œuvre globale de l'humanité. Le rapport qui unit l'individu à l'humanité est celui de la dérivée à l'intégrale, comme dans la monadologie leibnizienne.

Humboldt propose une approche sémiologique élargie, qui ne se réduit pas à l'étude du langage comme représentation conceptuelle. Sa démarche résolument non métaphysique ouvre sur une anthropologie de la culture qui admet une pluralité d'universaux. II revalorise les cultures extra-occidentales en montrant que les langues des peuples extra-occidentaux sont aussi complexes que les nôtres ${ }^{\mathbf{1 7}}$. Les peuples extra-occidentaux sont selon lui nos «égaux», même s'il lui arrive de donner sa préférence aux langues à flexion plutôt qu'aux langues agglutinantes. Humboldt conserve en effet une dimension téléologique dans son approche, ce qui peut donner à première vue une impression d'européano-centrisme, mais il tend en réalité à un dépassement de cette attitude, car c'est d'un processus universel de libération qu'il s'agit: l'individu doit aller par sa formation progressive vers une forme de liberté plus grande et s'élever à la compréhension de l'humanité,
15. Ernst Cassirer

souligne ainsi la filiation de Humboldt à Goethe: " "Toutes les figures se ressemblent, et aucune n'est égale aux autres. Et ainsi leur chœur guide vers une loi cachée", dit Goethe dans son poème "La métamorphose des plantes". Si dans ces vers nous substituons aux plantes les différents types de langage humain, nous avons une claire et concise expression du problème énoncé par Humboldt:

"Toutes les langues se ressemblent, et aucune n'est égale aux autres; et ainsi leur chœur guide vers une loi cachée" " (voir Rosenkranz 2007 : 315, trad. de l'auteur). Or chez Claude Lévi-Strauss, on trouve un enchaînement d'idées similaires, bien que Cassirer ne soit pas mentionné: "Si un résultat [se dégage de notre entreprise globale], c'est d'abord que nul mythe ou version de mythe n'est identique aux autres et que chaque mythe, en paraissant insister gratuitement sur un détail insignifiant et s'y appesantir sans raison avouée, cherche en fait à dire le contraire de ce que dit à ce sujet un autre mythe: aucun mythe n'est semblable. Pourtant, pris dans leur ensemble, ils reviennent tous à la même chose et, comme Goethe l'affirme des plantes, "leur chœur guide vers une loi cachée" " (voir Lévi-Strauss 2009 [1971] : 619-620). Si l'influence de Goethe sur Lévi-Strauss a été soulignée par de nombreux commentateurs, comme Jean Petitot (voir 1999: 97-122), il reste encore à relire Semper comme un des maillons de cette chaîne morphologique.

16. Voir le commentaire sur Humboldt que fournit André Stanguennec (2008: 92). La forme de la langue est le corrélat de l'acte constituant de la parole: "Abstraite de cet acte, la forme devient la "forme vide" qu'un certain structuralisme risquerait de considérer comme un résultat sans genèse. " $\mathrm{La}$ forme interne de chaque langue est « la forme concrète d'un acte, visée par et dans l'activité de parler elle-même».

17. Revalorisation que l'on retrouve chez Semper 2007: 306.

\section{ci-contre}

fig $=3$

Joseph Roques, «Tableau de la forme des principales feuilles ", in Plantes usuelles, indigènes et exotiques, dessinées et coloriées d'après nature, avec la description de leurs caractères distinctifs et de leurs propriétés médicales. Paris: Chez l'auteur, rue des fillesSaint-Thomas et chez Madame veuve Hocquart, 1807-1808, vol. I, pl. IV. Bibliothèque universitaire Claude Bernard Lyon 1. 
18. Nous soulignons.

19. Voir Trabant 1992: 177 Voir le chapitre décisif sur le caractère qui clôt l'ouvrage p. 166 notamment.

20. On désigne par Petites mythologiques les œuvres tardives de Lévi-Strauss telles que La Voie des masques, La Potière jalouse et Histoire de Lynx, trois textes qui figurent dans CEuvres, Vincent Debaene, Frédéric Keck, Marie Mauzé et Martin Rueff (éd.), Paris, Gallimard, 2008. quelle que soit sa culture. Le processus de configuration suit un mouvement de libération, sauf dans des moments de crise, où l'on perd le sens de la créativité de nouvelles formes.

On retrouve chez Semper des échos du projet de Humboldt dans ce passage décisif du début de la première partie du Style:

L'art possède son langage spécifique fait de types formels et de symboles qui se transforment de manière très variée au cours de l'histoire de la culture de telle sorte que, dans la manière qu'il a de s'exprimer à travers eux, règne une diversité presque aussi grande que celle qui existe dans le domaine de la langue particulière. Ainsi, de même que la linguistique la plus récente [sous-entendu celle de Humboldt] s'attache à mettre en évidence les relations d'affinité qui existent entre idiomes humains, à remonter le courant de la transformation des mots au fil des siècles en les ramenant à quelques sources vers laquelle ils convergent en une forme primitive commune, réussissant de la sorte à élever la philologie au rang de science authentique, à faciliter même la simple étude pratique des langues et à jeter sur le sombre territoire de l'histoire primitive des peuples une lumière inattendue, un effort de même nature se justifie dans le champ de l'histoire de l'art, consacrant cette attention qui lui appartient en propre à expliquer le développement des formes artistiques à partir de leurs germes et de leurs racines, leurs changements et ramifications ${ }^{\mathbf{1 8}}$. (Semper 2007 : 305)

Semper reprend en outre à Humboldt sa théorie de l'histoire, travail incessant d'individus qui comprirent leur temps et trouvèrent la formule juste pour satisfaire aux exigences de celui-ci. Cette formule pourrait sembler proche de la théorie hégélienne du grand homme. Or il n'en est rien car selon Semper, comme selon Humboldt: «L'individu qui anime», reste, «eu égard à la hiérarchie des éléments, [...] la pièce qui doit être prise en considération en premier » (ibid. : 231), et non l'État.

Semper emprunte également à Humboldt la notion récurrente de «caractère» (ibid.: 301), décisive pour comprendre le projet humboldtien de mise au jour des structures par comparaison des langues entre elles, tout en décrivant aussi les caractères propres à chaque langue, révélés dans l'acte vivant de parole. Structure et caractère d'une langue constituent les deux clés de voûte de son édifice. Il en va de même chez Semper, pour qui l'étude comparée des divers types d'architecture d'aires culturelles différentes est légitimée par «le pressentiment (épistémologiquement précaire) de leur caractère ${ }^{19}$ ».

Avant la lettre, Semper illustre à certains égards une anthropologie structurale aux accents morphologiques, comparable aux Petites mythologiques de Lévi-Strauss ${ }^{20}$. Selon Semper:

L'architecte qui sait reconnaître la signification la plus originelle des plus vieux symboles de sa propre langue et qui se rend compte de la manière dont avec l'art lui-même ils se transforment au cours de l'histoire [...] tire un profit comparable à celui que la linguistique comparative fig. 4

Ronan \& Erwan Bouroullec, Twigs and Algues, 2004

(C) Paul Tahon et R \& E

Bouroullec. 


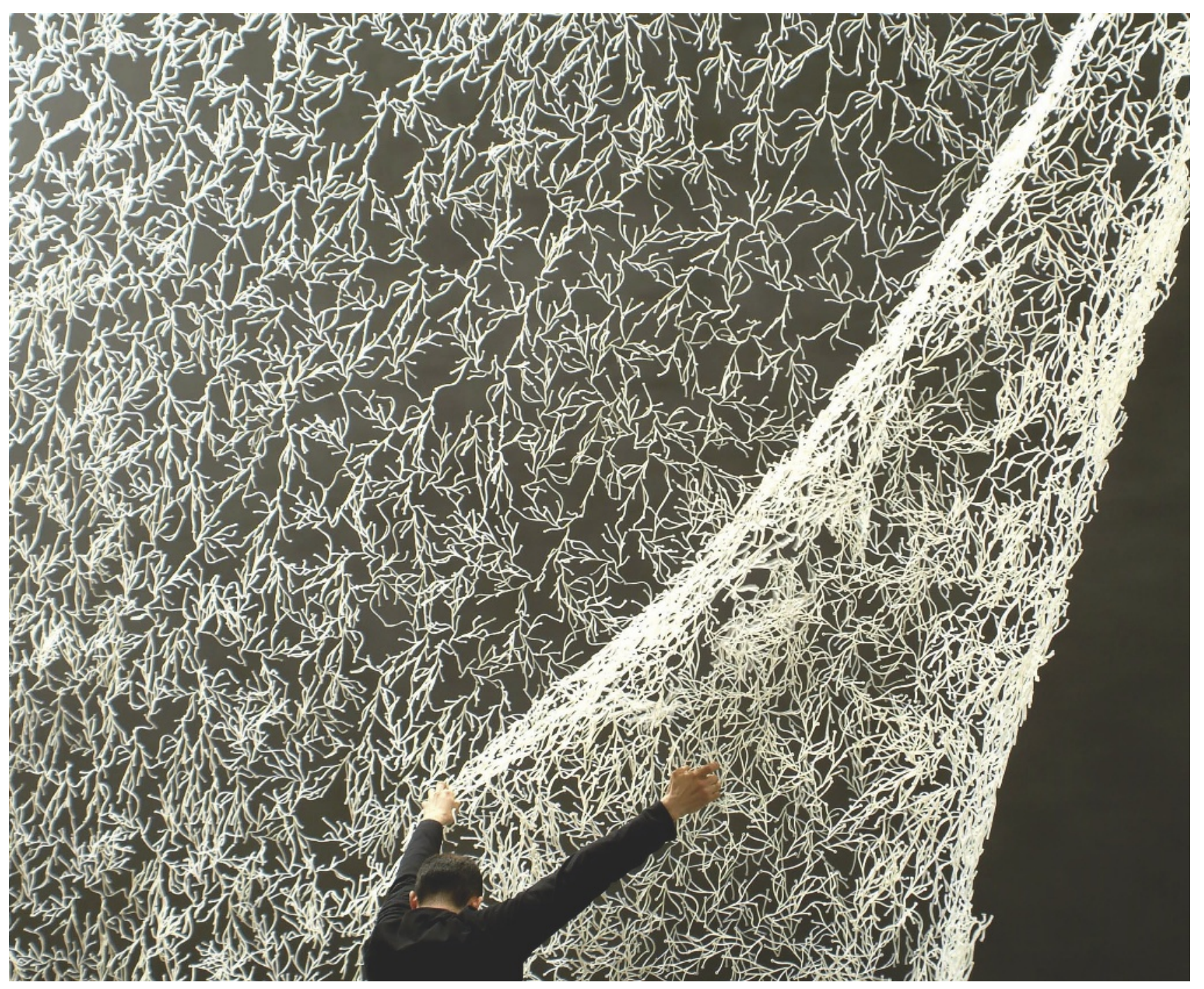


21. Voir Bourdieu 1972 chapitre II, "La maison ou le monde renversé »: 45 sq

22. Les dates de vie de Georg Simmel sont 1858 1918, tandis que Gottfried Semper naît en 1803 et meurt en 1879. Georg Simmel appartient donc à une autre génération que celle de Semper tout en restant un de ses contemporains. et la mise en évidence d'une communauté originaire des langues apportent à l'orateur moderne. Et je crois que l'époque n'est plus loin où la recherche des formes linguistiques et cette autre recherche qui s'intéresse aux formes artistiques entreront toutes les deux en interaction d'une manière telle que les éclaircissements réciproques les plus remarquables devront en résulter dans les deux domaines. (Semper 2007: 310)

Semper ne parle certes pas ici stricto sensu d'homologies structurales entre langage et art, comme ce sera le cas chez Lévi-Strauss qui aborde conjointement séries de mythes et séries de masques, mais il parle de réseaux d'analogies entre, par exemple, les parures d'un casque assyrien et les palmettes d'un bâtiment relevant de la même configuration du savoir (ibid. : 252).

C'est ce réseau d'analogies qu'explorera à sa manière Erwin Panofsky dans Architecture gothique et pensée scolastique (1967). Une ligne de Semper semble même résumer l'ouvrage à venir de Panofsky: «L'édifice gothique était la transcription lapidaire de la philosophie scolastique des $X \|^{\Theta}$ et XIII siècles. 》 (Semper 2007 : 281) Au croisement de considérations sociologiques et architecturales, c'est cet ouvrage de Panofsky qui donnera au sociologue Pierre Bourdieu l'idée de thématiser les forces formatrices d'habitude caractérisant ce qu'il va désigner comme l'habitus culturel spécifique de chaque entité sociale déterminée. Jouant le rôle de matrice, les études ethnologiques des déplacements dans l'espace symbolique de la maison kabyle ${ }^{21}$ conduiront Bourdieu à renouveler la compréhension de l'espace social, avec une approche qui n'est pas sans présenter quelques similarités avec les études sempériennes.

\section{Les positions sociologiques, pédagogiques et esthétiques de Simmel: un prolongement indirect de Semper}

D'un point de vue sociologique, l'auteur à convoquer pour comprendre Semper demeure toutefois Georg Simmel. Du fait de ses engagements politiques marqués et de son implication dans la vie sociale, liée à son métier d'architecte, Semper attache davantage d'importance à la dimension sociale des productions culturelles qu'un historien de l'art ou un théoricien de l'art ne pourraient le faire. Quels sont les points communs entre les conceptions de Semper et la sociologie formelle de Simmel ${ }^{22}$ ?

Tous deux abordent le problème de l'émergence de différents processus d'individualisation face à la multiplication des machines, la reproductibilité technique, la démultiplication des moyens modernes mis à disposition de l'individu et la modification du statut des matériaux résultant de l'arrivée de nouvelles techniques.

À la différence de Goethe et Humboldt, Simmel et Semper formulent un diagnostic critique de la situation de crise dans laquelle se trouve l'individu face à la culture. Ils prennent au sérieux le sentiment de démotivation potentielle de l'individu face à l'ampleur du matériau objectif qui se trouve face à lui (Semper 2007: 267). Simmel parle de «tragédie de la culture» (Simmel 1988: 177) pour évoquer cette tension entre l'individu et la grande 
masse d'objets, de techniques et de moyens mis à sa disposition: comment donner l'impression que tout cela est assimilable, pour redynamiser la créativité des individus? Lisons ces mots de Semper, que l'on retrouvera dans les analyses de Simmel: «L'époque présente n'a le temps ni de se reconnaître au milieu de cette abondance de bienfaits presque imposés, ni de les maîtriser. » (Semper 2012: 63) Et plus loin, dans un texte contemporain de l'Exposition universelle de Londres: «L'excès de moyens, tel est le premier danger sérieux contre lequel l'art va avoir à lutter. Cette expression est peut-être illogique (il n'y a pas de surabondance de moyens, mais une insuffisance dans le pouvoir de les maîtriser), toutefois, elle se justifie en ce qu'elle montre bien l'état de renversement de nos relations " (ibid. : 66).

On note ici un accent tragique, qu'on lit aussi chez Nietzsche, quand il analyse l'inversion des valeurs empêchant la créativité et le grand style. On retrouve plus tard encore cette tonalité tragique chez Warburg dans les dernières lignes de son exposé sur le rituel du serpent (2003: 133) et plus amplement chez Walter Benjamin, lorsqu'il analyse l'art à l'ère de la reproductibilité technique et la «perte d'aura » ainsi entraînée (2003 [1936]).

La dialectique bienheureuse qui animait forme et vie semble perdre de son dynamisme, analysent Semper et Simmel. Au lieu d'une véritable renaissance des formes originaires permettant à un nouveau style d'émerger et de s'installer dans la durée pour fructifier à nouveau ultérieurement, on assiste, selon Semper, à la production de copies mécaniques qui font perdre le sens même des types originaires.

II faut donc de manière urgente restaurer les moyens de se cultiver, de se former, et proposer à la fois une synthèse unificatrice, une vaste anthropologie, ambitieuse, intégratrice, qui permette de réorganiser la masse du matériau et, ce faisant, éviter surtout de couper cette enquête de la pratique concrète. L'érudition ne tient qu'à condition de demeurer créatrice, c'est-à-dire au service direct de la pratique.

Semper et Simmel ont en commun de penser aux solutions pédagogiques concrètes qui pourraient dénouer la tragédie en simple drame. Ils n'en restent pas à un conflit pessimiste concernant ce que d'autres désignent comme le «déclin de l'Occident » (Spengler 1948). Leur conclusion consiste à repenser de fond en comble la formation individuelle (la Bildung de l'individu).

Semper et Simmel analysent tous deux à leur manière ce que Simmel nomme les effets de socialisation sur l'organisation de l'espace (Simmel 2013 [1908]), avec des approches concrètes qui rappellent De l'esprit des lois de Montesquieu.

Enfin, bien que cela semble plus anecdotique à première vue, Semper et Simmel soulignent tous deux l'importance du cadre des œuvres picturales et de l'ornementation que doit de préférence porter ce cadre, en fonction de la taille du tableau, de son thème... Semper thématise l'importance du cadre (2007: 287) dans Le Style, en 1860. Le cadre permet la clôture, l'encerclement, la fermeture sur soi de ce qui va alors pouvoir être considéré comme 

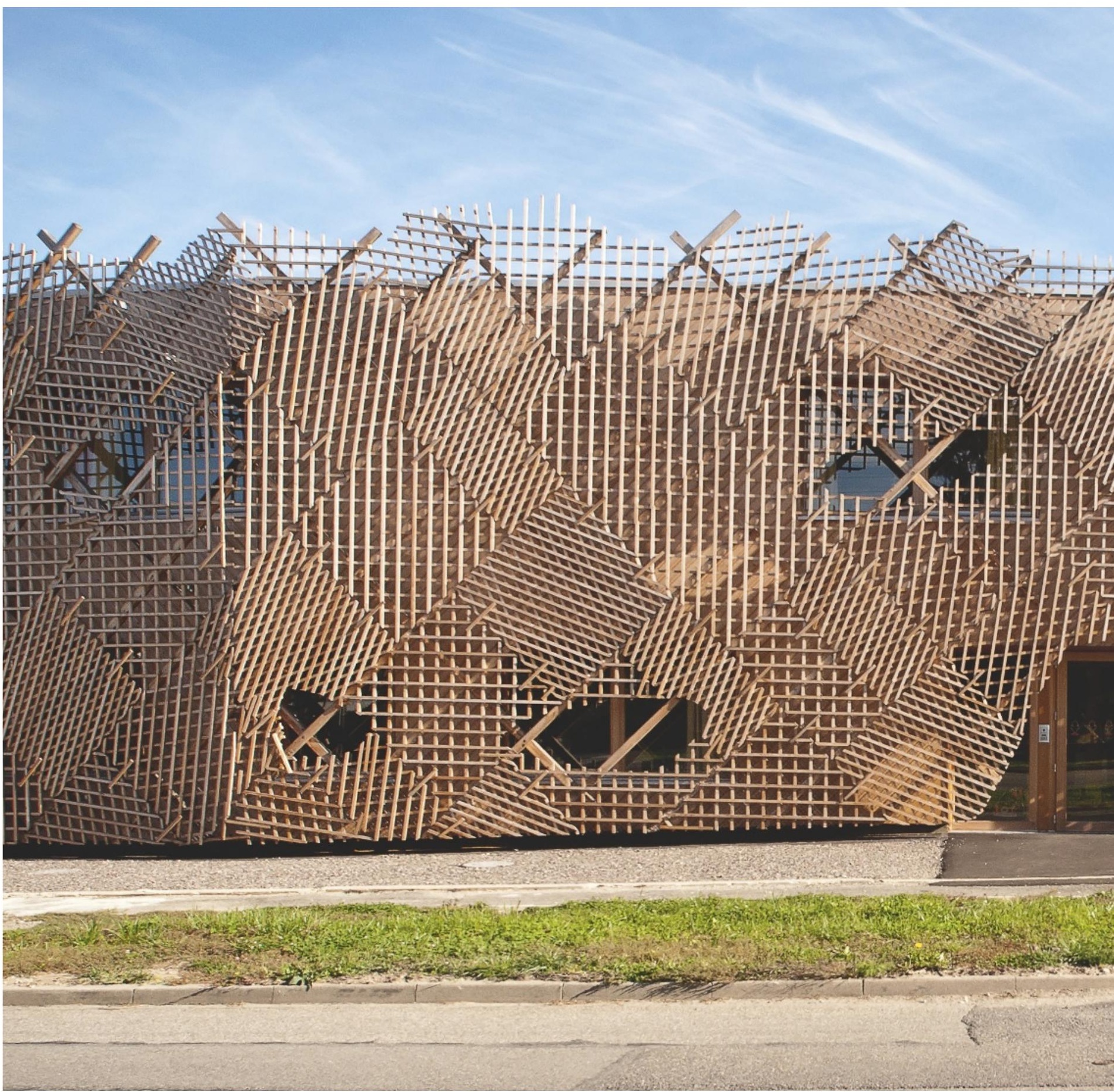


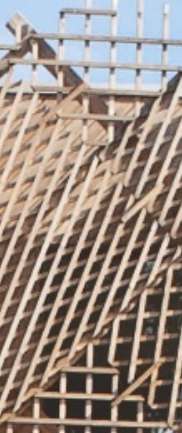




\section{ci-contre}

fig. 6

Aby Warburg, L'Atlas Mnémosyne, pl. 7

1921-1929. Pathos

du vainqueur. Triomphe

romain. Arc de triomphe.

Nikê. Apothéose

(ascension réussie).

Empereur = Dieu. Butin

(Gemma Augustea).

Élévation sur le bouclier.

Apothéose sous forme

d'élévation sur le

bouclier chez Napoléon.

Piétinement par les

chevaux. Couronnement.

Char montant vers le

ciel, symbole du soleil.

Soumission (Province).

Tête entre les mains.

(Légende d'après L'Atlas

Mnémosyne. Paris,

L'écarquillé-INHA, 2012,

p. 82). le centre d'une disposition eurythmique (ibid.: 288). Mais ce qui est décisif, c'est que Semper met en rapport le cadre avec la porte et la fenêtre qui, dans une architecture, encadrent la "personne qui se met en avant ou qui observe» (ibid.). Le cadre est «une des formes de base parmi les plus importantes en art. Pas d'images enfermées, ni d'échelle de grandeur sans lui. Ce n'est que par le cadre que l'eurythmie entre en application, et que la disposition régulière et concentrique des composantes formelles viennent à former une figure fermée autour de l'objet encadré» (ibid.). De même, la tenture ou le revêtement d'un mur est le symbole de la séparation spatiale nécessaire à l'émergence d'une figure (ibid.: 177). Simmel parlera lui aussi du cadre dans un petit essai daté de 1902, Le Cadre (2003 [1902]). Le cadre fait selon lui du tableau une île et, pour cela, son ornementation doit être de préférence perpendiculaire au tableau. Le cadre ne doit pas faire preuve d'individualité, mais de style, avance-t-il encore (ibid.: 37). Il doit témoigner d'une certaine neutralité pour contrebalancer l'individualisation dont l'œuvre fait, quant à elle, preuve. Pour que l'œuvre soit originale, individuelle, il faut paradoxalement qu'elle se détache, se découpe, s'autonomise d'un arrièreplan plus global, tout en pouvant, à un second niveau, être reconnectée aux autres œuvres avec lesquelles elle entre en série et en réseau selon un maillage complexe dont elle condense le principe. Il en est de même de l'individu, qui doit pouvoir se couper de la mode et dépasser les cercles sociaux intermédiaires qu'il constitue autour de lui, s'il veut à la fois être luimême et s'élever à l'idéal de l'humanité. Le cadre, à la fois présent et effacé, doit servir "d'intermédiaire entre l'œuvre d'art et son milieu ", qu'à la fois il relie et il sépare, une "tâche à laquelle dans l'histoire, l'individu et la société s'épuisent mutuellement » (ibid. : 40).

\section{Semper et la lecture qu'en feront Aby Warburg, Ernst Cassirer et Edgar Wind}

Un lien fort unit la pensée d'Aby Warburg à celle de son prédécesseur Semper. Tous deux sont portés par l'attention qu'ils accordent au détail. Semper s'intéresse par exemple à la disposition précise des feuilles d'une couronne et mène cette patiente description pour montrer que c'est à ce niveau et à ce niveau seul, celui du détail, à première vue invisible, que l'on peut comprendre ce qui rend un motif vivant. Warburg détaille de la même façon le pli d'un vêtement, l'ondoiement d'une chevelure, comme Semper parle de la coiffure et du drapé (Semper 2007: 241-242). Warburg a lu et annoté l'essai sempérien de 1856 "Sur les principes formels de la parure et leur signification comme symboles de l'art » (Semper 1856). II s'intéresse comme lui à l'ornementation non du point de vue de formes géométriques désincarnées, mais de celui de l'incarnation du mouvement dans le monde (Papapetros 2013). II relève avec enthousiasme la classification que fait Semper des ornements en trois catégories: le pendentif, ornement macrocosmique qui incarne le mouvement d'oscillation; l'anneau, ornement microcosmique qui délimite le rapport qu'entretient chaque partie du corps avec les autres, symbole des formations sociales; et enfin l'ornement de direction (Richtungsschmuck) qui accentue la direction du mouvement du corps de celui qui le porte et reflète son caractère ainsi que son expression. Warburg va reprendre sa thèse de la transformation du mouvement en ornement et son approche de l'origine dynamique de l'ornement corporel (ibid.). 


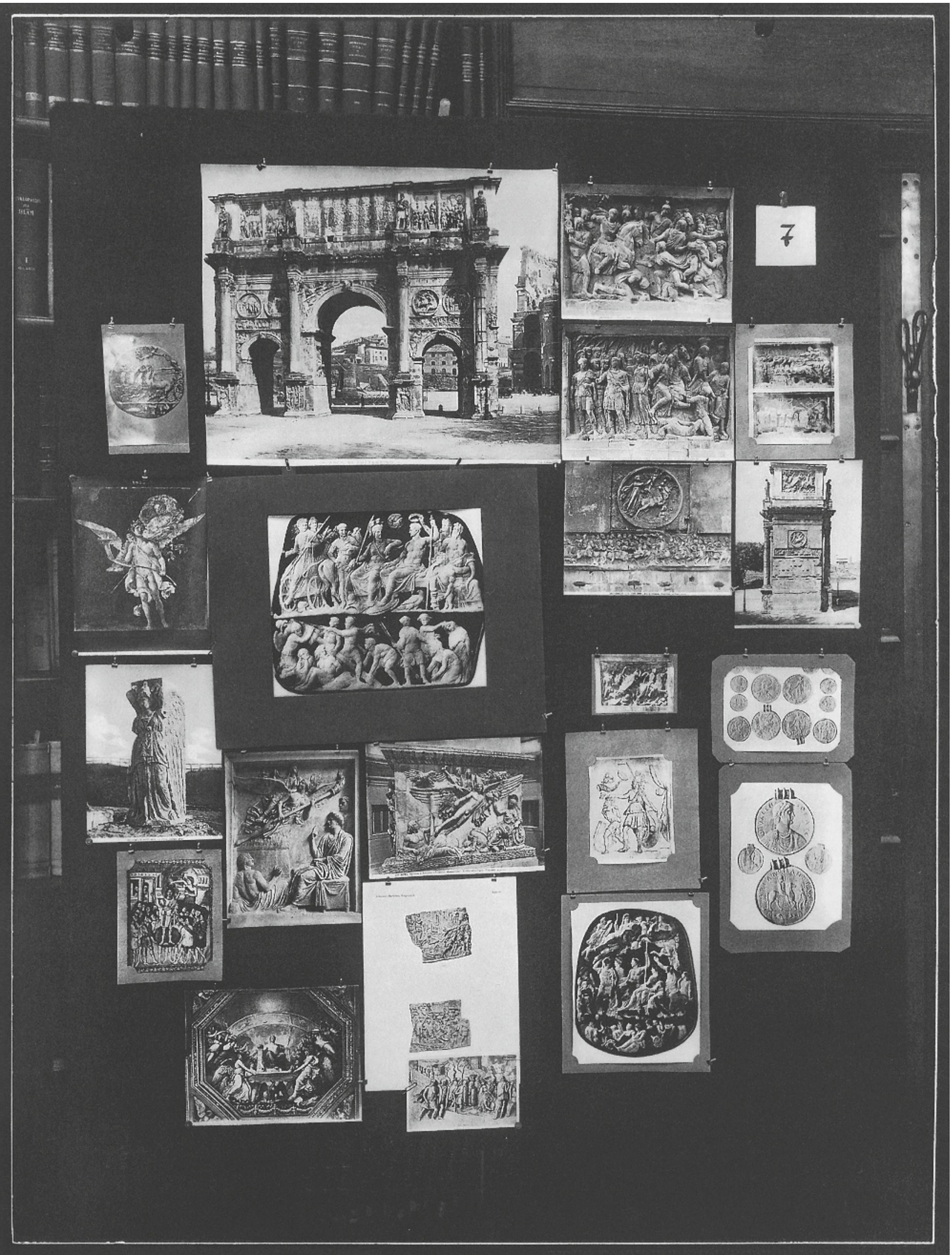


23. Voir Semper 2007: 309 : "Ces types proviennent de différents arts techniques du temps où ils étaient perçus dans leur utilisation la plus primitive [...]. S'ils acquièrent très tôt une signification symbolique [...], leur utilisation technique et spatiale la plus originelle ne disparut cependant jamais totalement et ils continuèrent bien plutôt à agir en tant qu'agents actifs de transformations postérieures des formes architecturales »; 310 : il précise la manière inconsciente dont cela fonctionne.

24. Concernant l'anonymisation des formes originaires dont est issue la culture et notamment l'architecture, voir Pinotti 2015: 31-40.

25. Voir Nietzsche 1988 135, § 149: "La lente flèche de la beauté - L'espèce de beauté la plus noble est celle qui ne ravit pas d'un seul coup, qui ne procède point par assauts fougueux et grisants (celle-là provoque facilement le dégoût), mais qui s'insinue lentement, que l'on emporte avec soi comme sans la sentir et qu'il nous arrive un jour de retrouver en rêve, mais qui, après avoir longtemps occupé une place modeste dans notre cœur, finit par prendre entièrement possession de nous, remplit nos yeux de larmes, ce cœur de nostalgie."
Est par excellence symbole ce qui exerce une activité souterraine inconsciente ${ }^{23}$. On ne prête habituellement pas attention au revêtement coloré d'une paroi, or c'est justement cela qui est l'essentiel car, selon Semper, c'est là que se joue une réminiscence confuse d'anciens modes de vie et de pensée et que les formes originaires du tissage et de la vannerie nous parviennent, malgré toutes les transformations culturelles subies. Le symbole est ce qui réunit I'homme primitif, et ses cultes magiques irrationnels, et l'homme technique contemporain, capable de modeler les matériaux de manière plastique et libre. Le symbole est ce trait d'union qui rassemble tous les hommes ${ }^{\mathbf{2 4}}$, il restaure soudain un état éloigné et procure une nostalgie créatrice très émouvante. «L'ornement garantit la survivance de la vie» (Papapetros 2013: § 37). On reconnaît ici la conception nietzschéenne de la «lente flèche de la beauté ${ }^{25}$ », qui fonctionne par la survivance de motifs et d'instincts et par la capacité à revivre des affects: imiter les Anciens pour faire survivre des instincts premiers (Nachleben et Nacherleben), qu'on libère des mécanismes asservissants qui ont pesé sur les hommes du passé.

Les planches de l'Atlas Mnémosyne constitué par Aby Warburg pour élaborer une grammaire visuelle des gestes pathétiques (2012) témoignent d'une revalorisation des arts mineurs, déjà présente chez Semper, pour faire «vibrer» les arts majeurs. Les planches de Warburg se polarisent et se repolarisent continûment, sous l'effet de l'organisation dynamique de groupes et de séries d'œuvres autour de figures complexes, matrices jouant le rôle de cas prégnants. De même que les arts techniques se trouvent ordonnés autour de l'art libre que constitue l'architecture chez Semper, les petites céramiques, les sceaux-cylindres, les instruments primitifs encadrent sur les planches de Warburg les œuvres centrales, chefs-d'œuvre que ces œuvres mineures font vibrer, leur donnant un sens profond. Le spectateur, interloqué, mis en mouvement, en vient à ne plus savoir si le plus décisif pour organiser les planches d'images est constitué par l'œuvre centrale, “expliquée» par ce qui l'entoure, «dépliée » pour s'y exprimer, ou au contraire par les œuvres mineures disposées autour du chef-d'œuvre. Ces œuvres mineures sont au fond peut-être en un sens l'essentiel, puisque c'est souterrainement, grâce à ces œuvres mineures relevant de l'art appliqué, que la liberté du chef-d'œuvre central apparaît, par oscillation réglée. Le chef-d'œuvre libère ses forces par ce léger dérangement qu'induit la proximité d'œuvres mineures habituellement passées sous silence. Nous pouvons en donner un exemple avec la planche 7 de l'Atlas (ibid. : 82-83) où figurent une reproduction photographique de l'Arc Constantin (en haut), aux côtés de médaillons en or et d'un Grand Camée de France, agrandis. L'œuvre architecturale prend corps par les arts appliqués qui l'entourent. Et cela produit un sentiment "d'inquiétante étrangeté " au sens freudien car ce qui n'était a priori pas animé s'anime miraculeusement. Il en va de même dans les études sempériennes de l'ornement, du revêtement, du décor, des parures.

Semper et Warburg sont tous deux à ce titre intéressés par les fêtes et les cérémonies (Semper 2007: 331). Alors qu'elles ne sont souvent traitées par les historiens de l'art que de manière anecdotique, elles donnent selon eux leur signification symbolique aux figures, comme survivance des liens vivants entretenus avec un groupe culturel. 
On peut souligner une autre proximité encore entre Semper et Warburg. Leur intérêt pour le phénomène d'expression se caractérise toujours par un certain retrait du lyrisme et du romantisme, par contraste avec des approches que l'on pourrait qualifier d'idéalistes. L'expression n'est pas thématisée à partir du seul pôle que constitue le sujet, mais plutôt et surtout à partir du pôle que constitue l'objet, en tant qu'il condense un processus de configuration ${ }^{26}$. Ainsi, Semper aborde des aspects qui pourraient intéresser aussi un romantique, mais il s'y intéresse avec le regard plus neutre et plus objectif de l'appareil photographique ou de la caméra, un décalage qui le rend particulièrement «moderne».

Enfin, l'idée de concevoir un musée idéal, ne cédant en rien à l'approche esthétisante d'un André Malraux mais s'enracinant au contraire solidement dans une théorie de la culture ambitieuse tout en servant la créativité à venir, est commune à Warburg et Semper.

Semper et Cassirer, philosophe allemand qui fut l'élève de Simmel et l'ami proche de Warburg, cheminent pourrait-on dire entre une approche morphologique goethéenne et une approche structuraliste «avant la lettre», telle qu'elle se déploiera chez Lévi-Strauss. Semper et Cassirer ont en commun de refuser tout réductionnisme. Ils revendiquent une approche complexe des œuvres d'art qui intègre de multiples niveaux de signification. L'approche morphologique s'ouvre sur une théorie de la culture élargie, définie comme anthropologie de l'homme en mouvement, selon les termes employés par Warburg ${ }^{27}$ pour décrire conjointement son projet et celui de Cassirer.

Cassirer veut saisir les aires relationnelles de projection ${ }^{\mathbf{2 8}}$ où se réfractent différentes formes symboliques, irréductibles entre elles, pour caractériser chaque époque culturelle. II développe une philosophie relationnelle qui aborde les formes symboliques comme des fonctions corrélées.

Or l'architecture apparaît explicitement chez Semper comme l'aire de projection commune des différents arts appliqués, vecteurs symboliques dont la logique propre est en droit irréductible à celle qui gouverne les autres: céramique, tissage, menuiserie, maçonnerie. L'étude de l'architecture devient le meilleur moyen de mettre au jour différentes configurations de savoirs, en dégageant l'idée propre à chaque aire culturelle.

Quant au rapport de Semper à l'historien de l'art et philosophe qu'est Wind (2012), lui-même élève de Warburg, de Cassirer et de Panofsky, il se tisse sur plusieurs points centraux de leurs esthétiques, en particulier la valorisation de la technique, de l'instrument et de l'outil. Je cite Semper: «Le matériau à traiter, l'informel d'où surgira une forme, sont également liés à l'instrument et à la main qui le manie » (Semper 2007 : 344). On peut souligner la grande modernité de cette approche qui lie forme, technique et style. Semper rappelle le lien étymologique entre le style et le burin, «instrument dont les Anciens se servaient pour écrire et dessiner » (ibid.). Parler de l'instrument et du geste pour le manier permet de penser la forme comme un résultat, connecté souterrainement aux gestes anciens de réponse à des besoins primaires. Un essai de Cassirer daté de 1931 sur le rapport de la forme
26. Ibid. : 303, où on retrouve les expressions de «régularité », «type», "caractère", «expression ».

27. Voir Cassirer 2009: 67. Lettre du 15 avril 1924 d'Aby Warburg à Ernst Cassirer: "Si nous voulons tous deux inaugurer "une science générale de la culture comme théorie de l'homme en mouvement". " (Trad. de l'auteur.)

28. Voir Cassirer 2001 : 9, avant-propos. II s'agit de « déterminer pour ainsi dire une aire relationnelle commune sur laquelle se projettent de manière identique, les évolutions religieuse, philosophique et littéraire, afin de mettre en évidence aussi bien ce qu'elles ont de spécifique dans leurs lois propres que le système universel des rapports que ces domaines entretiennent entre eux. " 


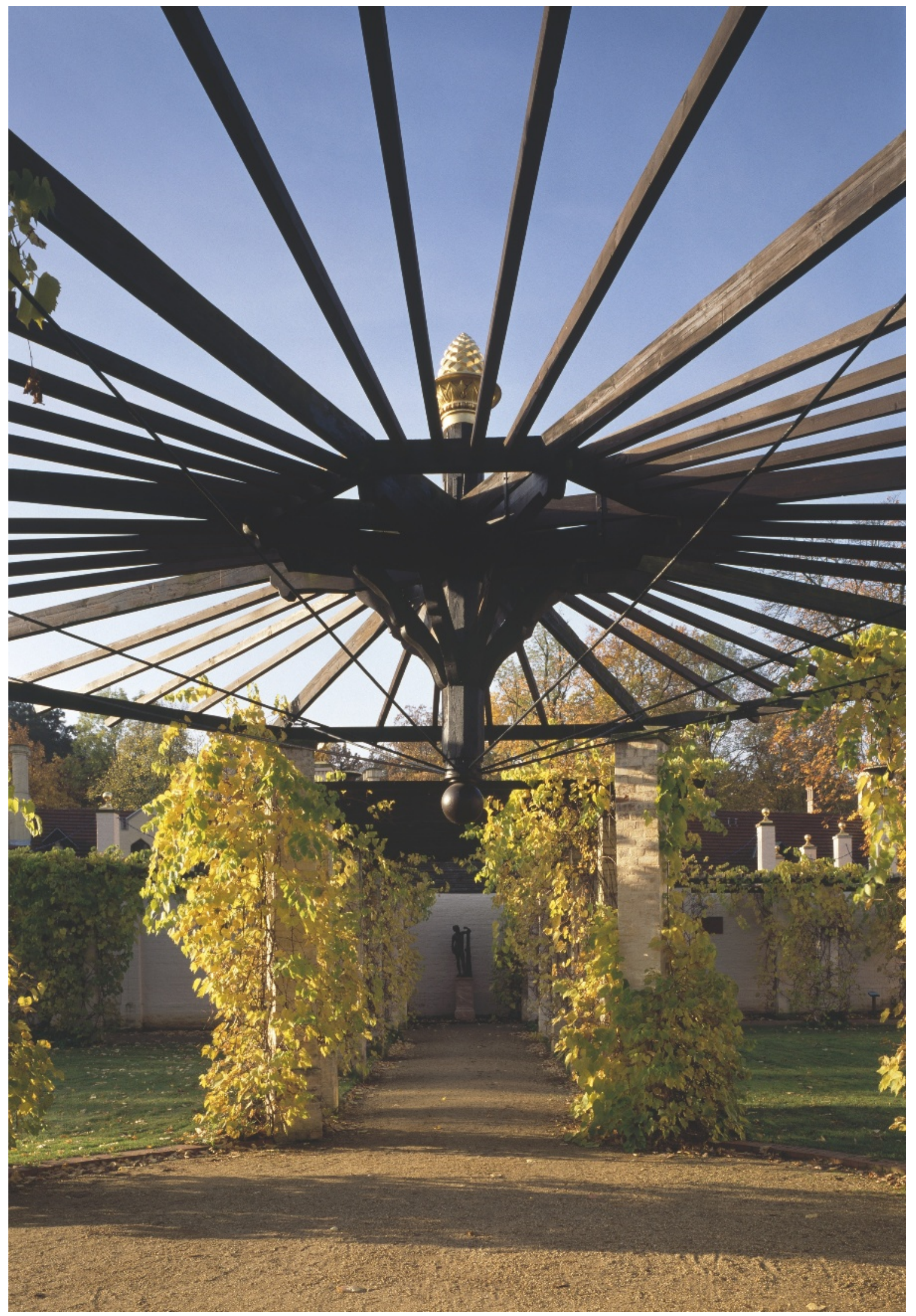


à la technique ${ }^{29}$ est révélateur de l'avènement de cette nouvelle approche revalorisant l'appareil et l'instrument qui trouvera sa formule chez Benjamin, mais aussi chez André Leroi-Gourhan et Gilbert Simondon. Wind défend une approche pragmatiste, au sens que l'expression prend chez Charles Sanders Peirce (et non pas en un sens «utilitariste», comme si la valeur se réduisait à la fonction naturelle, l'usage basique, le besoin). La conception de Wind est pragmatiste dans la mesure où elle part de l'agir, de l'action d'un corps dans un espace. Chez lui, l'esthétique se définit comme théorie de l'incorporation. Cette incorporation est double. D'une part l'utilisation d'un instrument, que ce soit un outil ou tout simplement le corps, permet à une théorie encore trop abstraite de prendre corps, de se réaliser, de se concrétiser. Mais d'autre part l'instrument est aussi incorporation des légalités nouvelles que fournissent les nouveaux objets que nous rencontrons et sur lesquels nous exerçons nos instruments. Ce n'est pas tant la capacité abstraite à schématiser le monde qui nous lie à lui que la capacité concrète à le mesurer, à le façonner par des outils et des instruments. Or, chez Semper, l'idée d'incorporation des symboles ou d'incarnation est décisive pour définir le style: l'œuvre d'art est le «résultat d'un processus», dont le style correspond à «l'élévation du thème fondamental à la signification artistique ainsi que de tous ses coefficients internes et externes, qui agissent à travers l'incarnation de ce thème transformé en œuvre d'art » (Semper 2007 : 264).

Nous voudrions conclure cette mise en perspective par la reconnaissance de l'originalité et de la modernité dont témoigne la pensée de Semper. En identifiant l'ornementation comme le symbole qui condense le principe caché ${ }^{30}$ permettant de créer des séries et des groupes de figures par transformation et en présentant un système d'analogies élargi qui intègre les pratiques de parures du corps en mouvement tout comme les diverses techniques de construction dans l'espace social et culturel, Semper offre un modèle morphologique d'approche des phénomènes artistiques très moderne. Son regard n'est pas le regard lyrique du romantique, mais le regard objectif qu'apportent plans, cartes, planches anatomiques, photographies ou prises de vues cinématographiques, bien qu'il soit assurément anachronique de se référer ici au film. Il aborde le plus objectivement possible les œuvres culturelles, tout en développant souterrainement une poétique discrète de l'organisme vivant, étonnant «Cuvier de la science de l'art ${ }^{\mathbf{3 1}}$ ». Partir de l'architecture et en montrer l'origine dans les activités de tissage, de façonnage du bois et de la cuisson de la céramique est le moyen d'ouvrir l'esthétique sur une anthropologie de la culture. Une approche qui aurait pu demeurer stérile et datée si elle s'était cantonnée à une esthétisation réductrice du phénomène artistique et si elle n'avait pas réussi, comme elle l'a au contraire fait et continue à le faire aujourd'hui, à susciter tout à la fois une réflexion générale sur la culture et un diagnostic critique du contemporain, ouvrant sur une définition humaniste du symbole comme trait d'union entre les hommes dits «primitifs» et ceux d'aujourd'hui.

CEPA (Culture, Esthétique et Philosophie de l'Art), Paris I vanvliet.muriel@gmail.com
29. Voir Cassirer 1995: 61, "Forme et technique».

30. Voir Semper 2007 : 346: "L'ornement constitue le premier pas significatif vers l'art. En lui et dans sa légalité est contenu le codex intégral de l'esthétique formelle. "

31. Andreas Hauser, "Der "Cuvier der Kunstwissenschaft". Klassifizierungsprobleme in G. Sempers Vergleichende Baulehre", in Thomas Bolt et Adolf Reinle, Grenzbereiche der Architektur, Basel/ Berlin/Boston, Birkhaüser, 1985: 97-114.

\section{ci-contre}

fig. 7

La pergola, au parc du château de Branitz à Cottbus édifié par Gottfried Semper. Photo Florian Monheim. Akg-images / Bildarchiv Monheim. 


\section{Benjamin, Walter}

2003 [1936] L'CEuvre d'art à l'époque de sa reproductibilité technique, trad. de l'allemand par Lionel Duvoy. Paris, Allia.

\section{Bourdieu, Pierre}

1972 Esquisse d'une théorie de la pratique précédé de Trois études d'ethnologie kabyle. Paris, Librairie Droz.

\section{Cassirer, Ernst}

1975 Essai sur l'homme, trad. de l'anglais par Norbert Massa. Paris, Les Éditions de Minuit.

1995 Écrits sur l'art, trad. de l'anglais par Christian Berner, Fabien Capeillères, Jean Carro et Joël Gaubert. Paris, Éditions du Cerf.

2001 Liberté et Forme: l'idée de la culture allemande, trad. de l'allemand par Jean Carro, Martha Willman-Carro et Joël Gaubert. Paris, Éditions du Cerf.

2009 Nachgelassene Manuskripte und Texte, t. XVIII, Ausgewählter wissenschaftlicher Briefwechsel, John Michael Krois, Marion Lauschke, Claus Rosenkranz et Marcel Simon-Gadhof (éd.). Hambourg, Felix Meiner Verlag.

\section{Goethe, Johann Wolfgang von}

1982 Poésies: du voyage en Italie jusqu'aux derniers poèmes, trad. de l'allemand par Roger Ayrault. Paris, Aubier-Montaigne.

1986 Traité des couleurs, trad. de l'allemand par Henriette Bideau. Laboissière-en-Thelle, Triades.

1996 Écrits sur l'art, trad. de l'allemand par Jean-Marie Schaeffer. Paris, Flammarion.

2013 La Métamorphose des plantes et autres essais botaniques, trad. de l'allemand par Henriette Bideau. Laboissière-en-Thelle, Triades.

\section{Humboldt, Wilhelm von}

1903-1936 «Einleitung zum Kawi-Werke" in A. Leitzmann (éd.) Gesammelte Werke, t. VII. Berlin, Akademie. (Introduction à l'œuvre sur le kawi, trad. de l'allemand par Pierre Caussat. Paris, Seuil, 1974).

\section{Lévi-Strauss, Claude}

1962 "La logique des classifications totémiques", in La Pensée sauvage. Paris, Plon.

2009 [1971] Mythologiques, t. IV (L'Homme nu). Paris, Plon.

\section{Nietzsche, Friedrich}

1988 Humain trop humain: un livre pour esprit libres I, suivi de fragments posthumes (1876-1878) Giorgio Colli et Mazzino Montinari (éd.), trad. de l'allemand par Robert Rovini. Paris, Gallimard.

\section{Papapetros, Spyros}

2013 «Warburg, lecteur de Semper ornement, parure et analogie cosmique ", trad. de l'anglais par Marie Sanquer et Anika Schwarzwald, Images Re-vues hors-série 4 [en ligne], disponible sur: http:// imagesrevues.revues.org/2862

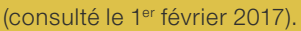

\section{Panofsky, Erwin}

1967 Architecture gothique et pensée scolastique, trad. de l'anglais par Pierre Bourdieu. Paris, Les Éditions de Minuit.

\section{Petitot, Jean}

1999 «La généalogie morphologique du structuralisme " Critique 620-621: 97-122.

\section{Pinotti, Andrea}

2016 «Why Anonymizing the History of Image? ", in Diego Cavalotti, Federico Giordano et Leonardo Quaresima (dir.), A History of Cinema without Names. Mimésis International.

\section{Riegl, Aloïs}

2002 [1893] Questions de style: fondements d'une histoire de l'ornementation, trad. de l'allemand par Henri-Alexis Baatsch et Françoise Rolland. Paris, Hazan.

\section{Rosenkranz, Claus (éd.)}

2007 Aufsätze und kleine Schriften, 1941-1945, Ernst Cassirer Gesammelte Werke, t. XXIV. Hambourg, Felix Meiner Verlag.

\section{Semper, Gottfried}

1856 «Über die formelle Gesetzmässigkeit des Schmuckes und dessen Bedeutung als Kunstsymbol ", Monatsschrift des wissenschaftlichen Vereins in Zürich 1: 101-130. Texte réédité in Kleine Schriften, recueil de textes posthumes réunis par Manfred et Hans Semper. Berlin/Stuttgart, Spemann, 1884 : 304-343.

2007 Du style et de l'architecture: écrits 1834-1869, trad. de l'allemand par Jacques Soulillou et Nathalie Neumann. Marseille, Parenthèses.

2012 Science, industrie et art. Estelle Thibault (éd.), trad. de l'allemand par Émile Reiber. Gollion, Infolio.

\section{Simmel, Georg}

1988 La Tragédie de la culture et autres essais, trad. de l'allemand par Sabine Cornille et Philippe Ivernel. Paris, Rivages.

2003 [1902] Le Cadre et autres essais, trad. de l'allemand pa Karine Winkelvoss. Paris, Gallimard.

2013 [1908] Sociologie: étude sur les formes de la socialisation, trad. de l'allemand par Sybille Muller. Paris, PUF.

\section{Spengler, Oswald}

1948 Le Déclin de l'Occident: esquisse d'une morphologie de I'histoire universelle, 2 t., trad. de l'allemand par Mohand Tazerout. Paris, Gallimard.

\section{Stanguennec, André}

2008 Être, soi, sens: les antécédences herméneutiques de la dialectique réflexive. Villeneuve d'Asca, Presses universitaires du Septentrion.

\section{Trabant, Jürgen}

1992 Humboldt ou le sens du angage, trad. de l'allemand par François Mortier et Jean-Luc Évard. Bruxelles, Mardaga.

\section{Warburg, Aby}

2003 Le Rituel du serpent: récit d'un voyage en pays pueblo, trad. de l'allemand par Diane H. Bodart, Philip Guiton et Sybille Muller. Paris, Macula.

2012 Atlas Mnémosyne, Roland Hecht (éd.), trad. de l'allemand par Sacha Zilberfarb. Paris, L'écarquillé/ INHA (Der Bilderatlas Mnemosyne, Martin Warnke et Claudia Brink [éd.] Berlin, Akademie Verlag, 2000).

\section{Wind, Edgar}

2012 Ästhetischer und kunstwissenschaftlicher Gegenstand: ein Betrag zur Methodologie der Kunstgeschichte, Pablo Schneider (éd.). Hambourg. Philo Fine Art.

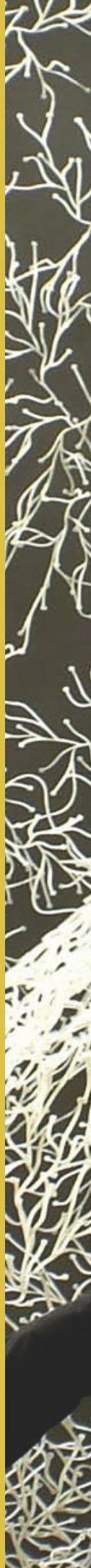

page 152 et ci-contre Ronan \& Erwan Bouroullec Twigs and Algues, 2004 (c) Paul Tahon et R \& E Bouroullec. 

+

a) (1) A N 54 (1) (1)

N(t)

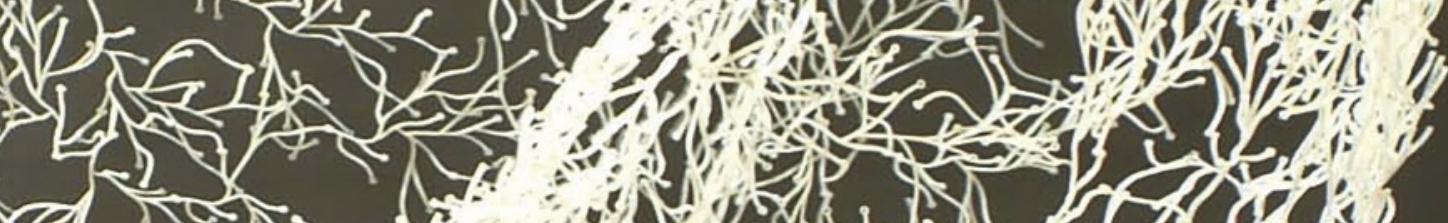
$4(2)$

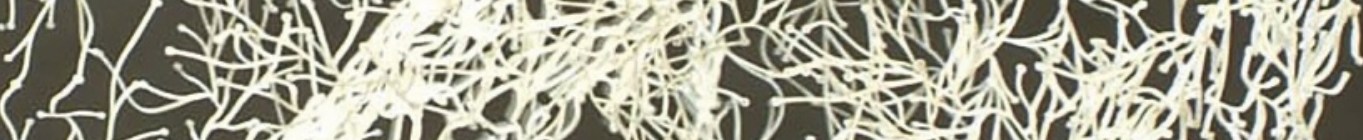

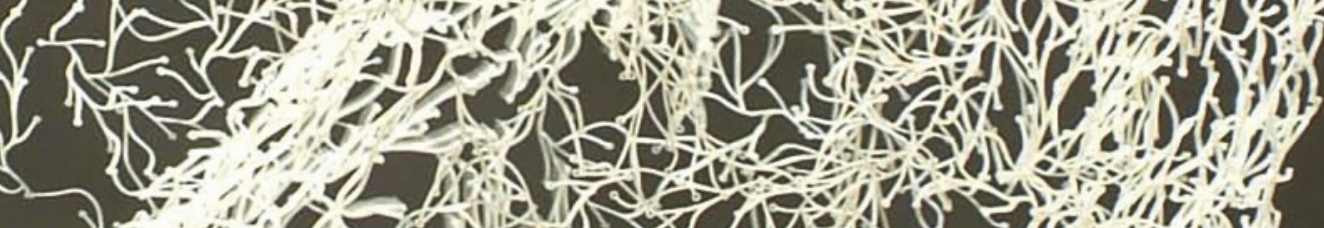

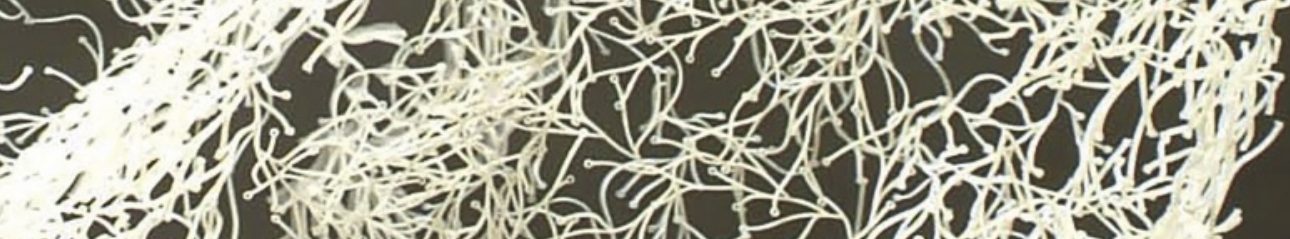
a

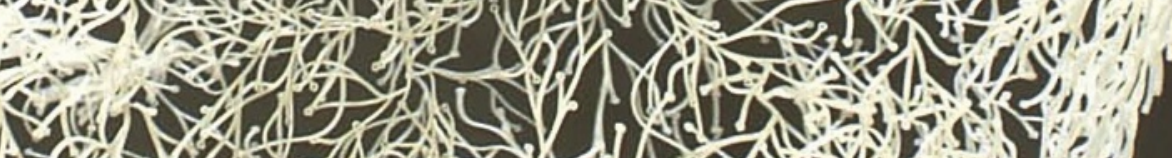

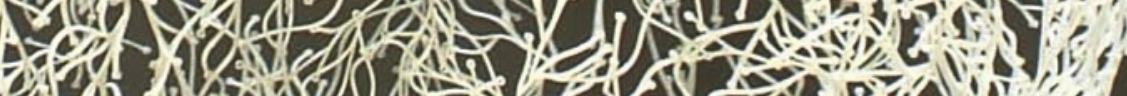
8 (1) 7.7.

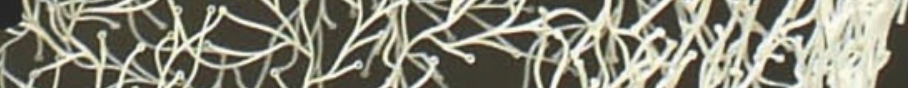

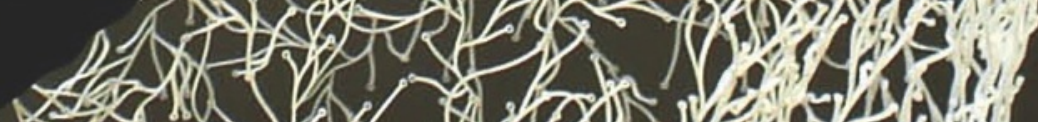

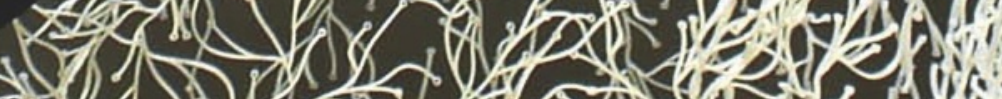
$\left\{\begin{array}{l}3 \\ \text { a }\end{array}\right.$ (1) (19) 